\title{
Efficient quantum processing \\ of three-manifold topological
}

\section{invariants}

\author{
S. Garnerone ${ }^{1,2,4}$, A. Marzuoli ${ }^{3}$ and M. Rasetti ${ }^{1,2}$
}

${ }^{1}$ Dipartimento di Fisica, Politecnico di Torino, corso Duca degli Abruzzi 24, 10129 Torino, Italy

garneron@usc.edu

${ }^{2}$ Institute for Scientific Interchange, Villa Gualino, Viale Settimio Severo 75, 10131 Torino, Italy annalisa.marzuoli@pv.infn.it

${ }^{3}$ Dipartimento di Fisica Nucleare e Teorica, Università degli Studi di Pavia and Istituto Nazionale di Fisica Nucleare, Sezione di Pavia, via A. Bassi 6, 27100 Pavia, Italy

mario.rasetti@polito.it

\begin{abstract}
A quantum algorithm for approximating efficiently three-manifold topological invariants in the framework of SU(2) Chern-Simons-Witten (CSW) topological quantum field theory at finite values of the coupling constant $k$ is provided. The model of computation adopted is the $q$-deformed spin network model viewed as a quantum recognizer in the sense of [1], where each basic unitary transition function can be efficiently processed by a standard quantum circuit.
\end{abstract}

e-print archive: http://lanl.arXiv.org/abs/quant-ph/0703037

${ }^{4}$ Department of Physics and Astronomy and Center for Quantum Information Science \& Technology, University of Southern California, Los Angeles, CA 90089, USA 
This achievement is an extension of the algorithm for approximating polynomial invariants of colored oriented links found in [2,3]. Thus all the significant quantities - partition functions and observables — of quantum CSW theory can be processed efficiently on a quantum computer, reflecting the intrinsic, field-theoretic solvability of such theory at finite $k$.

The paper is supplemented by a critical overview of the basic conceptual tools underlying the construction of quantum invariants of links and three-manifolds and connections with algorithmic questions that arise in geometry and quantum gravity models are discussed.

\section{Introduction}

The possibility of computing quantities of topological or geometric nature was recognized as a major achievement for quantum information theory in a series of papers by Michael Freedman and co-workers [4-6] (see also [7] for an introduction). Their "topological quantum computation" setting, intrinsically fault-tolerant and protected from decoherence, was designed to comply with the behavior of "modular functors" of 3D Chern-SimonsWitten (CSW) non-abelian topological quantum field theory (TQFT) [8-10], the gauge group being typically SU(2). In physicists' language, such functors are partition functions and correlators of the quantum theory and, owing to gauge invariance and invariance under diffeomorphisms, which freeze out local degrees of freedom, they share a global, "topological" character. More precisely, the physical observables are associated with topological invariants of knots - the prototype of which is the Jones polynomial [11] — and the generating functional is an invariant of the three-dimensional ambient manifold, the Reshetikhin-Turaev-Witten invariant $[8,12]$.

The search for efficient quantum algorithms to compute (approximations of) knot invariants has been carried out by several groups in the last few years.

Within the framework of topological quantum computation the existence of an effective procedure has been taken for granted by resorting to the fact that this model is polynomially reducible to the standard quantum circuit model [13]. Later on, this implicit proof has been supported by the fundamental notion of "additive approximation" introduced in [14] and borrowed in all the alternative approaches.

The first explicit algorithm for the Jones polynomial of a knot presented as the plat closure of a braid has been constructed in [15] by efficiently approximating unitary matrices associated with a particular representation of the braid group, whereas in [16] the knot presentation and the representation of 
the braid group are generalized (these notions will be defined in the following sections). It is worth stressing that both these approaches rely on the standard model of quantum computation, namely the quantum circuit model based on qubits, elementary unitary gates and related algorithmic techniques such as the so-called Hadamard trick [13].

The approach we proposed in $[2,3]$ differs from the previous ones in many respects. First, we were able to handle more general knot invariants, namely "colored" link polynomials (a link is a multicomponent knot) which reduces to Jones' in a particular case. Second, the model of quantum computation we adopted, that we refer to as the $q$-deformed spin network model, is in some sense a blending of the computational schemes mentioned above framed within the background provided by the theory of finite-states quantum machines [1]. This topic will be addressed in Section 2, while a few conceptual questions and implications of our model of computation will be discussed in the last section of this introduction.

In the main part of the paper, Section 3, we extend our efficient algorithm for approximating colored link polynomial to deal with "quantum" threemanifold invariants.

In order to overcome the difficulties due to the fact that we shall resort to concepts and definitions arising in many different contexts (low-dimensional topology, quantum group theory, CSW field theory, three-dimensional quantum gravity models, classical and quantum complexity theory), we shall illustrate in the next few paragraphs the basic conceptual tools underlying the construction of such "universal" invariants.

\subsection{From quantum topology to TQFT}

The term "quantum topology" was introduced by Turaev [17] to denote implications on the topological side of the algebraic theory of quantum groups - technically, deformations of universal enveloping algebras of Lie groups. The latter, based on the pioneering work of Drinfel'd [18] and Jimbo [19], was inspired by theoretical physics from its very beginning since quantum groups and associated $R$-matrix representations are the basic tools of quantum inverse scattering methods and are the backbone of exactly solvable models in statistical mechanics [20].

The deformation parameter $q$ was originally assumed to be a real number related to Planck constant by $q=e^{h}$, therefore it is commonly referred to as a "quantum" deformation, whereas the "classical", undeformed Lie group symmetry is recovered at the particular value $q=1(h \rightarrow 0)$. When dealing 
with quantum invariants of knots and three-manifolds $[17,21] q$ is most often a complex root of unity, the case $q=1$ being considered as the "trivial" one. However, in a topologist's language, "classical" topological invariants are not the $(q=1)$-counterparts of "quantum" invariants, but rather the usual invariants of algebraic and geometric topology, typically related to the fundamental group and homology groups of manifolds and submanifolds.

As Roberts remarks in the introduction to [21] the standard topological invariants were created in order to distinguish between things and, owing to their intrinsic definitions, it is clear what kind of properties they reflect. For instance, the Euler number $\chi$ of a smooth, closed and oriented surface $\mathcal{S}$ determines completely its topological type and can be defined as $\chi(\mathcal{S})=$ $2-2 g$, where $g$ is the number of handles of $\mathcal{S}$. On the other hand, quantum invariants of knots and three-manifolds were discovered, but their indirect construction based on quantum group technology often hides information about the purely topological properties they are able to detect.

What is lost at the topological level is however well paid back by the possibility of bridging this theory with a plenty of issues in pure mathematics and theoretical physics (cf. the review [21] and the list of references therein). To the early connections mentioned above (quantum inverse scattering, exact solvable models) it is worth adding the operator algebra approach used originally by Jones in defining his knot polynomial [11]. However, the most profitable development of the theory was that suggested by Schwarz and formalized by Witten [8] (see [22] for a review and original references).

Indeed, recognizing quantum invariants as partition functions and vacuum expectation values of physical observables in CSW TQFT provides a "physical" explanation of their existence and properties. Even more radically, one could speak of a "conceptual" explanation, as far as the topological origin of these invariants keeps on being unknown. In this wider sense, quantum topology might be thought of as the mathematical substratum of an $\mathrm{SU}(2)$ CSW topological field theory quantized according to the path integral prescription (the coupling constant $k \geq 1$ is constrained to be an integer related to the deformation parameter $q$ by $\left.q=\exp \left(\frac{2 \pi \mathrm{i}}{k+2}\right)\right)$.

The CSW environment provides not only the physical interpretation of quantum invariants but it does include as well all the historically distinct definitions [23]. In particular, monodromy representations of the braid group [24] appear in a variety of conformal field theories since point-like "particles" confined in two-dimensional regions evolve along braided worldlines ( [25] and references therein). As a matter of fact, the natural extension of CSW theory to a three-manifold $\mathcal{M}^{3}$ endowed with a non-empty two-dimensional boundary $\partial \mathcal{M}^{3}$ induces on $\partial \mathcal{M}^{3}$ a specific quantized boundary conformal 
field theory, namely the SU(2) Wess-Zumino-Witten (WZW) theory at level $\ell=k+2[8,26]$. The latter provides in turn the framework for dealing with $\mathrm{SU}(2)_{q}$-colored links presented as closures of oriented braids and associated with Kaul unitary representation of the braid group [27,28]. A further extension of this representation proposed by the same author in [29] is used in this paper to construct explicitly the quantum three-manifold invariants in the form originally defined in [30] within a purely algebraic setting. Such quantities are essentially the Reshetikhin-Turaev-Witten invariants [12] evaluated for three-manifolds presented as complements of knots/links in the threesphere $S^{3}$, up to an overall normalization.

\subsection{Algorithmic complexity of CSW theory}

As mentioned in the introductory remarks, the "quantum field" computer [4-6] is a model of computation designed to process quantities of topological nature arising in CSW environment and thus "efficiency" of any calculation - such as that of Jones knot polynomial - should be guaranteed by definition (we leave aside here the issue of "exact" versus "approximate" calculation at least for the moment).

However, when dealing with algorithmic questions, the model of computation adopted should comply with the commonly accepted paradigms of theoretical computer science. Turing machines, together with the polynomially equivalent circuit models based on elementary boolean gates, represent the universal schemes which allow problems and algorithms to be grouped into classical complexity classes [31]. In quantum computing, the notions of quantum Turing machine - and associated quantum circuits based on qubits and unitary elementary gates - can be introduced and represent the standard, universal model of computation [13].

It was shown in [5] that SU(2) CSW functors at the fifth root of unity, whose domain is restricted to collections of "topological" qubits (disks with three marked points) on which suitable unitary representations of the braid groups $\mathbf{B}_{3}$ and $\mathbf{B}_{3} \times \mathbf{B}_{3}$ act, do reproduce the standard elementary gates of the quantum circuit model. The physical support of such information processing consists of anyonic systems obeying particular types of braid statistics and work is in progress on the experimental side to check the implementability of such approach (see [32] and also [33] for a different kind of implementation of braiding operators).

Based the above properties, a sort of "minimal" realization of the full quantum field computer is (polynomially equivalent to) the quantum circuit 
and indeed the work of $[15,16]$ on quantum computation of the Jones invariant does not depend at all on any field theoretic background. Generally speaking, this is satisfactory for the aim of introducing an ad hoc computing model for treating anyonic quantum systems; yet is somehow disturbing because classical Turing machines and their probabilistic counterparts are able to simulate efficiently only any dynamical systems governed by classical laws at any degree of accuracy. Even the objection that we are in the presence of a quantum field theory - not simply a quantum mechanical many-body system - is misleading since the CSW model is exactly solvable at the full quantum level (for each fixed value of the coupling constant) without resorting to any approximation such as the low-energy limit $[22,23]$. The crucial feature of possessing only global, purely topological degrees of freedom makes quantum CSW theory likely to be simulated within a computational scheme based on a discrete space of states and able to implement polylocal braiding operations.

As will be illustrated in Section 2, the universal model of computation able to handle all discrete, many-body quantum systems described by (real or virtual) pure angular momenta states (not simply two-level systems) as well as solvable field theories of CSW-type is the ( $q$-deformed) spin network proposed in $[2,3]$.

As already recognized in [4] the task of computing the Jones polynomial of a knot or link represents a major achievement since it is the simplest observable in quantum CSW theory and then its calculation is a testing ground of the effectiveness of topological quantum computation. However, the interest in this algorithmic problem has recently grown in connection with the search for new testing grounds for quantum information theory in general, without necessarily exploiting the physical meaning of the invariant.

The reason why Jones link polynomial is so crucial in the computational context relies on the fact that a "simpler" link invariant, the AlexanderConway polynomial, can be computed efficiently, whereas the problem of computing two-variable polynomials - such as the HOMFLY invariant - is NP-hard (see $[21,34,35]$ for definitions of these invariants, original references and accounts of algorithmic questions).

The issue of computational complexity of the one-variable Jones polynomial in classical information theory can be summarized in

Problem 1.1. How hard is it to determine the Jones polynomial $J(L, q)$ of a link $L$ ?

A quite exhaustive answer has been provided in [35], where the evaluation of the Jones polynomial of an alternating link $\tilde{L}$ at a root of unity $q$ is 
shown to be \#P-hard, namely computationally intractable in a very strong sense.

Recall first that "alternating" links are special instances of links, the planar diagrams of which exhibit over and under crossings, alternatively. Thus the evaluation of the invariant of generic, not only alternating links is at least as hard. Secondly, the computation becomes feasible when the argument $q$ of the polynomial is a second, third, fourth, sixth root of unity, so that the first not easy case involves a fifth root of unity (refer to [35] for details on this technical issue). Finally, the $\# \mathbf{P}$ complexity class can be defined as the class of enumeration problems in which the structures that must be counted are recognizable in polynomial time. A problem $\pi$ in $\# \mathbf{P}$ is said to be $\# \mathbf{P}$-complete if for any other problem $\pi^{\prime}$ in $\# \mathbf{P}$, $\pi^{\prime}$ is polynomial-time reducible to $\pi$; if a polynomial time algorithm were found for any such problem, it would follow that $\# \mathbf{P} \subseteq \mathbf{P}$. A problem is $\# \mathbf{P}$-hard if some $\# \mathbf{P}$-complete problem is polynomial-time reducible to it. Other instances of $\# \mathbf{P}$-complete problems are the counting of Hamiltonian paths in a graph [31] and the more intractable problems arising in statistical mechanics, such as the enumeration of all configurations contributing to ground state partition functions [20].

The computational intractability of Problem 1.1 does not rules out the possibility of "approximating" efficiently Jones invariant, so that we may ask

Problem 1.2. How hard is it to approximate the Jones polynomial $J(L, q)$ of a link $L$ at a fixed root of unity $q(q \neq 2 n d, 3 r d, 4$ th, 6th root $)$ ?

Loosely speaking, the approximation we are speaking about is a number $Z$ such that, for any choice of a small $\eta>0$, the numerical value of $J(L, q)$, when we substitute in its expression the given value of $q$, differs from $Z$ by an amount ranging between $-\eta$ and $+\eta$ (see Section 3.2 below for a more precise statement). In the framework of classical complexity theory no algorithm to handle efficiently Problem 1.2 exists, while the answer in the quantum computational context was given in [14] (see also [16]):

The approximation of the Jones polynomial of a link presented as the closure of a braid at any fixed root of unity is $\mathbf{B P Q}-$-complete. Moreover, this problem is universal for quantum computation, namely is the "prototype" of all problems efficiently solvable on a quantum computer.

Recall that BQP is the computational complexity class of problems which can be solved in polynomial time by a quantum computer with a probability 
of success at least $\frac{1}{2}$ for some fixed (bounded) error. In [14] it was proved that $\mathbf{P}^{J}=\mathbf{B Q P}$, where $\mathbf{P}^{J}$ is defined as the class of languages accepted in polynomial time by a quantum Turing machine with an oracle for the language defined by Problem 1.2. This equality between computational classes implies that, if we find out an efficient quantum algorithm for Problem 1.2, then the problem itself is complete for the class $\mathbf{B Q P}$, namely each problem in this class can be efficiently reduced to a proper approximate evaluation of the Jones polynomial of a link [16].

Explicit, efficient quantum algorithms for approximating the Jones polynomial were proposed by Aharonov et al. [15] and by Wocjan and Yard [16], whereas an early attempt can be found in [36].

In our papers $[2,3]$ we proved that efficient algorithms can be implemented for approximating the larger class of "colored" Jones polynomials of links (addressed also in [37]).

The issue of colored link invariants brings us back to the quantum CSW environment, where they represent the most general gauge invariant physical observables of the theory, being vacuum expectation values of generic Wilson loop operators $[8,22,23]$. In Section 3 we shall provide a generalization of our algorithm for colored polynomials to handle the quantum three-manifold invariants introduced in Section 1.1.

Summarizing our results, we have shown that all the significant quantities - partition functions and observables - in SU(2) quantum CSW theory can be efficiently approximated at finite values of the coupling constant $k$. The intrinsic field-theoretic solvability of CSW theory is thus reflected by its computability on a quantum computer. Looking at the question the other way around, classical computational intractability of Jones and colored polynomials can be viewed as a consequence of their quantum nature. This feature has prevented up to now both exact and approximate efficient calculations of such topological quantities on classical (probabilistic) machines as it happens for simulations of any non trivial "genuine" quantum mechanical system (see, e.g., Feynman's proof in [38]).

\subsection{Quantized geometry versus quantum computing}

In this section we address some implications of our results in connection with algorithmic questions that may arise in other physical theories whose dynamical variables have a geometric character, typically quantum gravity models in $D=3$ and 4 space-time dimensions. 
We have however to face preliminarily a conceptual dilemma, namely whether

(i) an abstract universal model of computation, able to simulate any discrete quantum system including solvable topological field theories, must exists by its own

or

(ii) a (suitably chosen) quantum system is by itself a computing machine whose internal evolution can reproduce the proper dynamics of classes of physical systems.

The second alternative is becoming quite popular thanks to Lloyd's model, where a net of computing units generates a (superposition of) $4 D$ spacetimes [39] (see also [40] where similar ideas were anticipated).

The idea that many (if not all) aspects of our reality may be thought of as "outputs" of some kind of information processing is both appealing and intriguing. In this connection the role of information theory and its tools is so enhanced that it becomes a unifying paradigm. Of course, the classical version of hypothesis (i) is usually taken for granted as far as, on the one hand, a (probabilistic) Turing machine is capable of simulating the evolution of any classical system within a given accuracy, and, on the other, all concrete, finite-size realizations of the abstract machine obey the laws of classical physics. The practicability of hypothesis (ii) depends heavily on which system is chosen as a simulator and which types of boundary or initial conditions must be imposed to reproduce the dynamical behavior of observed physical systems. Moreover, the concept of "efficient" processing of information seems difficult to be handled without an abstract reference model of computation.

With the previous remarks in mind, we favour assumption (i), where the reference model of computation can be represented by the spin network simulator [41-43] (see also [44]) or its $q$-deformed, finite-size counterpart $[2,3]$ (see also Section 2 below). Note however that it may be tempting to assert that the spin network - thought of as a real net of interacting spin variables - can play as well the role of the reference quantum system in statement (ii). This is due to the fact that the recoupling theory of $\mathrm{SU}(2)$ angular momenta - representing the algebraic substratum of the simulator - is the main ingredient of the "spin network models" introduced by Ponzano and Regge [45] and Penrose [46]. Here classical, discretized 3D euclidean geometry arises from recouplings of quantum angular momenta in the asymptotic, large angular momentum limit (see [42], section 5 for a brief 
account). Thus, much in the sense of (ii), spin networks may act - under suitable constraints - as computing machines able to process information encoded into quantum spins to output "quantized" three-geometries obeying Einstein equations in the classical limit.

Thinking back to the issue of algorithms for quantum invariants defined in the framework of quantum CSW theory, our results can be used to test the algorithmic complexity of quantum gravity models in $D=3$ spacetime dimensions too. This achievement can be justified - independently from spin network models - by exploiting the close connection between CSW theory and $3 D$ gravity both as classical field theories and at the quantum level $[8,26]$ (classical $3 D$ gravity with a positive cosmological constant, reinterpreted as an $\mathrm{SU}(2)$ gauge theory, is quantized through the Euclidean path integral prescription).

For a closed orientable Riemannian three-manifold $\mathcal{M}^{3}$, let $Z\left(\mathcal{M}^{3} ; k\right)$ denote the Witten partition function associated with the classical CS action $S_{\mathrm{CS}}(A), A$ being the connection one-form. The functional $Z\left(\mathcal{M}^{3} ; k\right) \overline{Z\left(\mathcal{M}^{3} ; k\right)} \equiv\left|Z\left(\mathcal{M}^{3} ; k\right)\right|^{2}=\mathcal{Z}\left(\mathcal{M}^{3} ; k\right)$ for finite $k$ is the partition function of $3 D$ Euclidean quantum gravity in the first-order form, where the coupling constant $k$ is related to the cosmological constant $\Lambda$ by $k=4 \pi / \sqrt{\Lambda}{ }^{1}$

The point here is that every manifold in the class considered here can be presented as the complement of a link $L$ in the three-sphere (technically, by surgery along a framed version of $L$, see Section 3.1), so that $\mathcal{M}^{3} \equiv \mathcal{M}_{L}^{3} \sim\left(S^{3} \backslash L\right)$ and $\mathcal{Z}\left(\mathcal{M}_{L}^{3} ; k\right)=\left|Z\left(\mathcal{M}_{L}^{3} ; k\right)\right|^{2}$, where $Z\left(\mathcal{M}_{L}^{3} ; k\right)$ is the Reshetikhin-Turaev-Witten quantum invariant introduced in Section 1.1. Thus the results on algorithms for quantum invariants of links and threemanifolds summarized in Section 1.2 work equally well in the context of Euclidean $3 D$ quantum gravity models, reflecting once more the exact solvability of the theory for finite $k$.

An even more interesting connection between $\mathrm{SU}(2)_{q}$ quantum invariants and quantum gravity emerges when dealing with canonical quantization methods applied to general relativity in $(3+1)$ dimensions. We refer in particular to the "loop representation" based on Ashtekar "connection

\footnotetext{
${ }^{1}$ Note in passing that the invariant $\mathcal{Z}\left(\mathcal{M}^{3} ; k\right)$, on the one hand, equals the TuraevViro invariant for triangulated three-manifolds [47] and, on the other, can be derived by relating $3 D$ gravity to a $B F$-type topological field theory $[8,22,26]$. Recall that the name "BF theories" derives from the fact that their metric-independent classical action takes the form $\int B \wedge F_{A}$, where $F_{A}$ is the curvature of a connection $A$ and the $B$-field is a Lagrange multiplier. In contrast to Chern-Simons theory such topological field theories exist and are well defined in arbitrary dimensions.
} 
representation" of canonical gravity (see $[48,49]$ for reviews and original references). The Reshetikhin-Turaev-Viro invariants act there as quantum states associated with the boundary three-geometries (spatial slices of $4 D$ spacetimes). Such states were shown to satisfy both the quantum diffeomorphism constraint and the quantum Hamiltonian constraint (WheelerDeWitt equation) once a cut-off - related to the deformation parameter $q$ - has been fixed. Thus quantum invariants constitute the natural kinematical arena of loop quantum gravity [50]. This sketchy scenario hides however the necessity of introducing new types of quantum invariants the perturbative invariants - which arise when the cut-off of the underlying quantum field theory is removed.

In the CSW field-theoretic setting, perturbative invariants emerge as coefficients of the asymptotic expansion of the partition function $Z\left(\mathcal{M}_{L}^{3} ; k\right)$ as $k \rightarrow \infty$ (see [21, Chapter 7]). Besides a first term that corresponds to the semiclassical approximation of $Z\left(\mathcal{M}_{L}^{3} ; k\right)$ (saddle point), each contribution in the expansion is $\exp \left\{2 \pi k S_{\mathrm{CS}}(\hat{A})\right\}$ times a power series in $1 / k$ (here $S_{\mathrm{CS}}(\hat{A})$ is the CS action evaluated for the flat connection $\hat{A})$. Perturbative invariants are the coefficients of the powers $(1 / k)^{n}$ evaluated by using $(n+1)$-loop Feynman diagrams. No complete perturbative treatment of quantum CSW theory is available at present, and the meaning of such invariants in geometric topology is only conjectured in a few cases.

We are interested here in discussing briefly the "volume conjecture" concerning special classes of hyperbolic three-manifolds (recall that the volume is a topological invariant for such manifolds). Note also that most manifolds obtained by surgery on framed links in the three-sphere can be endowed with hyperbolic metrics. Focusing in particular on "hyperbolic knots", namely those knots which give rise to finite volume hyperbolic three-manifolds, the volume conjecture proposed in [51,52] (see also the review [21] for extended versions) can be cast in the form

$$
2 \pi \lim _{N \rightarrow \infty} \frac{\log \left|J_{N}(K)\right|}{N}=\operatorname{Vol}\left(S^{3} \backslash K\right)
$$

where $K$ is a hyperbolic knot and the notation $J_{N}(K)$ stands for the $N$-colored polynomial of $K$ evaluated at $q=\exp (2 \pi \mathrm{i} / N)$.

As pointed out many times, all quantum algorithms dealing with link polynomials are established for a fixed choice of the root of unity $q$ appearing in the argument of the invariants, while the volume conjecture involves the analysis of the asymptotic behavior of single-colored polynomials of a same knot for increasing values of the coloring itself. 
It would be interesting to explore the possibility of borrowing some of the techniques employed in [53] for dealing with the asymptotics of the Jones polynomial to test conjecture (1.1) within the computational framework for colored polynomials addressed in the present paper.

Note finally that the relevance of this issue for quantum gravity stems from the observation that all vacuum solutions of Einstein field equations in $(2+1)$ dimensions with a negative cosmological constant are hyperbolic metrics. As recently shown by Carlip [54], the smallest hyperbolic volumes give rise to the largest contributions in the saddle point term of the path integral of the quantum theory. This implies in turn that the so-called "real tunneling geometries" are most probable (a real tunneling geometry represents the transition from a compact Riemannian spacetime to a Lorentian one within the framework of the Hartle-Hawking "no boundary" approach to quantum cosmology [55]). On the other hand, the possibility of controlling such invariants from the algorithmic point of view might help also in selecting weights to be assigned to three-geometries in the "sum over topologies" within a "fully quantum" cosmology theory.

\section{$2 \quad$ Processing braiding operators}

\section{$2.1 \quad q$-Deformed spin network and quantum recognizers}

As pointed out in the introduction, our reference model of computation to deal with quantum topological invariants derived from Kaul unitary representations of colored oriented braids $[27,28]$ is given by the $q$-deformed spin network model.

The (undeformed) spin network simulator has been extensively addressed elsewhere [42] but for the convenience of the reader we have collected in Appendix A a few mathematical details. However, in order to recognize the necessity of introducing a $q$-deformed version, it is worth to discuss here the (categorical) foundations of the quantum theory of angular momenta. The computational space of the spin network simulator — modelled as a graph — encodes the representation ring of the Lie group $\mathrm{SU}(2)$ - namely finite-dimensional Hilbert spaces supporting irreducible representations (irreps) of $\mathrm{SU}(2)$ endowed with two binary operations, tensor product $\otimes$ and direct sum $\oplus$ (that provide a ring structure over the field $\mathbb{C}$ ) — together with all the unitary operators relating (multiple tensor products of) such spaces. Unlike the usual quantum circuit model [13], here it is possible to handle directly eigenstates of $N$ binary coupled angular momentum variables 
labelled by integers and half-integers $j_{1}, j_{2}, \ldots, j_{N}$ (in $\hbar$ units) and not simply $N$-qubit states labelled by the fundamental irrep $j_{1}=j_{2}=\cdots=j_{N}=$ $1 / 2$. The (re)coupling theory of $N \mathrm{SU}(2)$ angular momenta provides the whole class of unitary transformations that can be performed on many-body quantum systems described by pure angular momentum binary coupled sets of eigenstates [56, topic 12]. The unitaries that we need here (referred to as $j$-gates in Section 3 of [42]) are phase transformations, related to swaps of two contiguous spins and recoupling transformations expressed in terms of $3 n j$-coefficients of $\mathrm{SU}(2)$ and related to changes in the interaction schemes of the $N$ angular momenta $(N=n+1)$.

Within the framework of the categorical approach, the $\mathrm{SU}(2)$ representation ring is an instance of a unitary tensor category, endowed with intertwiner spaces and two basic morphisms, a "twist" (a trivial type of "braiding") and an "associator" [57,58]. The former acts on the tensor product of two Hilbert spaces $V, W$ supporting irreps of $\mathrm{SU}(2)$ by exchanging the order of the factors, namely

$$
\begin{aligned}
& R_{V, W}: V \otimes W \longrightarrow W \otimes V, \quad \text { with } \\
& R_{W, V} \circ R_{V, W}=\operatorname{Id}_{V \otimes W} .
\end{aligned}
$$

The explicit action of $R$ on a (binary coupled) state is a trivial phase transform, see (A.8), (A.9) in Appendix A.

The associator $F$ relates different binary bracketing structures in the triple tensor product of irreps $V, U, W$

$$
F:(V \otimes U) \otimes W \longrightarrow V \otimes(U \otimes W)
$$

and is implemented on a binary coupled state as a Racah transform involving one Racah-Wigner $6 j$-symbol (see (A.6), (A.7) in Appendix A). Note that both (2.1) and (2.2) are isomorphisms (unitary morphisms between intertwiner spaces in the categorical language) but the associator reflects a true (physically measurable) modification of the way in which intertwiner spaces are coupled.

The remarkable fact, derived from the general theory of braided tensor categories [59], is that more complicated multiple tensor products can be handled without introducing any further independent morphism. Actually, multiple tensor product spaces can be related by different combinations of braidings and associators, so that the basic morphisms must satisfy compatibility conditions, a so-called pentagon identity and two hexagon identities. In the more concrete language of $\mathrm{SU}(2)$ recoupling theory, each $3 n j$-symbol can be obtained as a combination of phase and Racah transforms and the 
non-uniqueness of such decomposition is translated into the BiedenharnElliott (pentagon) identity and the Racah identity (see, e.g., [56] or [60] for their explicit expressions).

In order to deal with non-trivial braiding operators - to be used in connection with the study of braid group representations and braid statistics (typically occurring in anyonic systems) — we are forced to modify the operation $R$ in (2.1) by introducing a genuine, non-trivial braiding morphism [59]

$$
\begin{gathered}
\mathcal{R}_{V, W}: V \otimes W \longrightarrow W \otimes V, \text { with } \\
\mathcal{R}_{W, V} \circ \mathcal{R}_{V, W} \neq \operatorname{Id}_{V \otimes W} .
\end{gathered}
$$

A consistent way of modifying the $\mathrm{SU}(2)$ tensor category to include nontrivial braidings can be achieved by moving to the representation ring $\mathfrak{R}$ $\left(\mathrm{SU}(2)_{q}\right)$ of the $q$-deformed Hopf algebra of the Lie group $\mathrm{SU}(2), \mathrm{SU}(2)_{q}(q$ a root of unity). The resulting braided tensor category is the "universal" algebraic structure underlying the constructions of quantum invariants of links and three-manifolds outlined in Sections 1.1 and 1.2. (We refer the reader to $[2,61]$ for short technical surveys of the quantum group and CSW approaches.).

According to the above remarks, it should be clear that the " $q$-deformed" spin network model of computation $[2,3]$ is modelled on the $q$-tensor category

$$
\left(\mathfrak{R}\left(\mathrm{SU}(2)_{q}\right) ; \mathcal{R} ; \mathcal{F}\right)
$$

where we have denoted by $\mathcal{F}$ the $q$-counterpart of the associator $F$ in $(2.2)$. Once suitable basis sets are chosen in the finite collection of irreducible spaces $\in \mathfrak{R}\left(\mathrm{SU}(2)_{q}\right)$, the unitary morphisms $\mathcal{R}$ and $\mathcal{F}$ can be made explicit. In particular $\mathcal{F}$ turns out to contain the $q$-deformed counterpart of the $6 j$-symbol and, regarding it as a unitary matrix, it is also referred to as "duality" (or "fusion") matrix borrowing the language of conformal field theories [25].

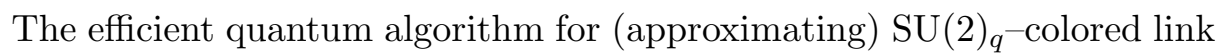
polynomials we obtained in [3] relies on a two-level procedure which can be summarized as follows:

- Kaul unitary representation of colored oriented braids - associated with links presented as plat closures of such braids - is processed on the $q$-spin network (2.4) in a number of steps that grows polynomially in the size of the input. In particular, each elementary computational step is implemented by applying either $\mathcal{R}$ or $\mathcal{F}$ (see Section 2.2 below). 
- The basic $q$-morphisms $\mathcal{R}, \mathcal{F}$ in the Kaul representation can be efficiently compiled on a standard quantum computer, by means of universal elementary gates acting on suitable qubit-registers. ${ }^{2}$

In order to analyze in more details the first topic above we need to frame the $q$-deformed spin network model within the theory of quantum automata and quantum languages. According to [1] a quantum recognizer is a particular type of finite-states quantum machine defined as a five-tuple $\{Q, \mathcal{H}, X, Y$, $\mathbf{T}(Y \mid X)\}$, where

1. $Q$ is a set of $\mathfrak{n}$ basis states, the internal states;

2. $\mathcal{H}$ is an $\mathfrak{n}$-dimensional Hilbert space and we shall denote by $\left|\Psi_{0}\right\rangle \in \mathcal{H}$ a start state expressed in the given basis;

3. $X$ and $Y=\{$ accept, reject, $\epsilon\}$ are finite alphabets for input and output symbols respectively ( $\epsilon$ denotes the null symbol);

4. $\mathbf{T}(Y \mid X)$ is the subset of $\mathfrak{n} \times \mathfrak{n}$ transition matrices of the form $\{T(y \mid x)=$ $U(x) P(y) ; x \in X, y \in Y\}$, where $U(x)$ is a unitary matrix which determines the state vector evolution and $P(y)$ is a projection operator associated with the output measurement on (suitable complete sets of observables associated with) the upgraded state vector.

In this kind of machine the output alphabet is chosen in such a way that a word $w$ written in the input alphabet $X$ must be either accepted or rejected, while for the null symbol the requirement is $P(\epsilon) \equiv \mathbb{I}$ (the identity matrix). Thus the one-step transition matrices applied to the start state $\left|\Psi_{0}\right\rangle$ can in principle assume the forms

(a) $T(\epsilon \mid x)=U(x) P(\epsilon) \equiv U(x) \mathbb{I} \forall x \in X$,

(b) $T$ (accept $\mid x)=U(x) P$ (accept) $\forall x \in X$ with $P$ (accept) $\equiv\left|\Psi_{0}\right\rangle\left\langle\Psi_{0}\right|$,

(c) $T$ (reject $\mid x)=U(x) P$ (reject) $\forall x \in X$ with $P$ (reject) $\equiv \mathbb{I}-\left|\Psi_{0}\right\rangle\left\langle\Psi_{0}\right|$,

according to whether no measure is performed (case (a)), or the output is "accept" / "reject", namely cases (b)/(c) respectively.

\footnotetext{
${ }^{2}$ This result is quite interesting by itself as recently pointed out in [62] where relations between the basic morphisms and large Fourier transforms are addressed.

The problem of finding out efficient algorithms to compute the fundamental functions of the quantum theory of $\mathrm{SU}(2)$ angular momenta - Clebsch-Gordan coefficients, $6 j$-symbols - has attracted much attention [63]. However, to our knowledge, there are neither classical nor quantum algorithms available for evaluating these functions for arbitrary values of their arguments. The crucial remark is that a $q-6 j$-symbol with arbitrary entries can be efficiently compiled and approximated on a quantum circuit independently of the input size of the algorithmic problem owing to the presence of the natural cut-off provided by $k$ (see Section 3.2, in particular footnote 8 ). Then we realize once more that the $q$-symmetry of solvable topological field theories is indissolubly tied with the effective computability of these models.
} 
The general axioms stated above can be suitably adapted to make this machine able to recognize a language $\mathcal{L}$ endowed with a word-probability distribution $\mathfrak{p}(w)$ over the set of words $\{w\} \in \mathcal{L}$. In particular, for any word $w=x_{1} x_{2} \ldots x_{l} \in \mathcal{L}$ the recognizer one-step transition matrix elements are required to be of the form $T_{i j}\left(x_{s}\right)=U_{i j}\left(x_{s}\right)$ on reading each individual symbol $x_{s} \in w$, namely no measurement is performed at the intermediate steps (here $i, j$ run from 1 to $\mathfrak{n}$, the dimension of the Hilbert space $\mathcal{H}$ ). Each $U_{i j}\left(x_{s}\right)$ must satisfy the condition

$$
\left|U_{i j}\left(x_{s}\right)\right|^{2}>0
$$

and the recognizer upgrades the (normalized) initial state to

$$
U(w)\left|\Psi_{0}\right\rangle \doteq U\left(x_{l}\right) \ldots U\left(x_{1}\right)\left|\Psi_{0}\right\rangle .
$$

Then the machine assigns to the word $w$ the number

$$
\mathfrak{p}(w)=\mid\left\langle\Psi_{0}\right| U(w) P(\text { accept }) U(w)\left|\Psi_{0}\right\rangle \mid \quad \text { with } 0 \leq \mathfrak{p}(w) \leq 1,
$$

which corresponds to the probability of accepting the word $w$ as a whole.

More generally, the machine accepts a word $w$ according to an a priori probability distribution $\operatorname{Pr}(w)$ with a word-probability threshold $0 \leq \delta \leq 1$ if

$$
|\operatorname{Pr}(w)-\mathfrak{p}(w)| \leq \delta, \quad \forall w \in \mathcal{L}
$$

In what follows the accuracy $\delta$ will be set to 0 , so that the two probability distributions $\mathrm{P} r$ and $\mathfrak{p}$ coincide.

\subsection{The Kaul representation as a quantum language}

In this section we shall show that the $q$-deformed spin network computational scheme (2.4) embodies families of quantum finite-states machines (or quantum automata) $\left\{\mathcal{A}_{q}\right\}$ - parameterized by the labels of $N$ irreps of $\mathrm{SU}(2)_{q}, q=$ root of unity - that recognize the language generated by the braid group according to a probability distribution given by the square of the modulus of the $q$-colored link polynomial.

This construction complies essentially with what we have done in [2], but here we should stress the interpretation of the Kaul representation [28] as 


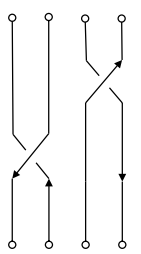

Figure 1: An oriented braid on four strands.

a quantum language, on the one hand, and the role played by the probability distribution, on the other (leaving aside details on the field-theoretic background material).

The basic ingredients of Kaul's construction are oriented geometric braids (see figure 1), the strands of which are endowed with "colorings" given by $\mathrm{SU}(2)_{q}$ irreps labels. An $n$-strand colored oriented braid is defined by two sets of $n$ assignments $\hat{j}_{i}=\left(j_{i}, \varepsilon_{i}\right)$ with $(i=1,2, \ldots, n)$, corresponding to the spin $j_{i}$ labelling the strand and to the orientation $\varepsilon_{i}$ of the strand, with $\varepsilon_{i}= \pm 1$ (for the strand going into or away, respectively, from a horizontal rod from which the braid issues). The first set of assignments is associated to the upper rod, the second to the lower rod (we use the convention that two braids are composed in the downward direction). The conjugate of $\hat{j}_{i}$ is defined as $\hat{j}_{i}^{*} \equiv\left(j_{i},-\varepsilon_{i}\right)$. It follows that the assignments on the lower rod are just a permutation of the conjugates of the assignments on the upper rod. A colored and oriented braid can thus be represented by the symbol

$$
\sigma\left(\begin{array}{llll}
\hat{j}_{1} & \hat{j}_{2} & \ldots & \hat{j}_{n} \\
\hat{l}_{1} & \hat{l}_{2} & \ldots & \hat{l}_{n}
\end{array}\right),
$$

where $\hat{l}_{j}=\hat{j}_{\pi(i)}$ for some $i$ and $j$ and a permutation $\pi$ of $\{1,2, \ldots n\}$.

The composition of two colored oriented braids is well defined only if the orientations and the colors of the two braids match at the merging points, as shown in figure 2 .

The group of colored oriented braid is generated by the identities (one for each assignment of colors and orientations on a topologically unentangled braid) and by the braids of type $\sigma_{l}$, as shown in figure 3 .

The collection of $\left\{\sigma_{l}\right\}$ for $l=1,2, \ldots n-1$ are the standard generators of the (colored) braid group $B_{n}$ and satisfy the following defining relations:

$$
\begin{gathered}
\sigma_{i} \sigma_{i+1} \sigma_{i}=\sigma_{i+1} \sigma_{i} \sigma_{i+1} \\
\sigma_{i} \sigma_{j}=\sigma_{j} \sigma_{i}, \quad|i-j| \geq 2 .
\end{gathered}
$$




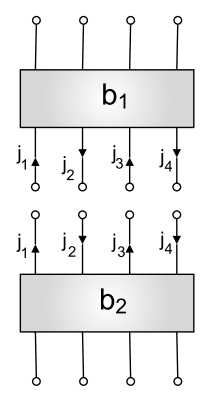

Figure 2: The composition of two colored oriented braids.

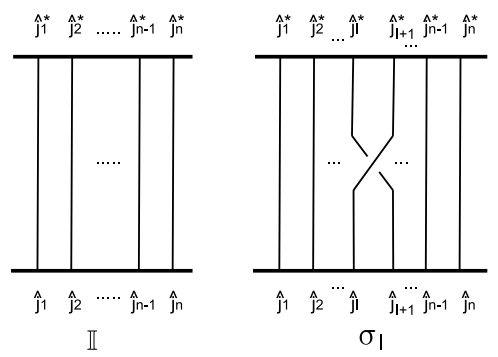

Figure 3: The identity and the generator $\sigma_{l}$ of the colored oriented braid group.
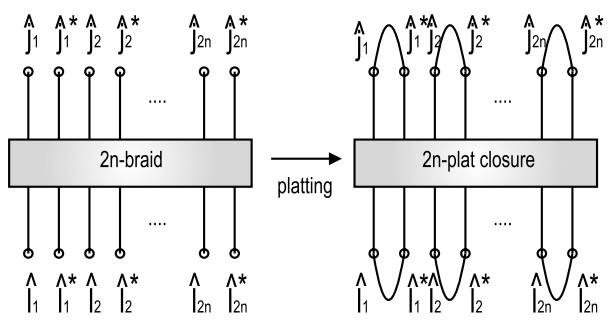

Figure 4: The platting of a colored oriented braid on $2 n$ strands.

The inverse of a generator $\sigma_{l},\left(\sigma_{l}\right)^{-1}$, corresponds to the under-crossing of the left strand $\hat{j}_{l}$ in figure 3 (right).

In order to obtain a link (multicomponent knot) from a colored braid we need to "close up" the braid. For our purposes we may consider only the plat closure (or platting) of a colored braid, defined for braids which possess an even number of strands and whose assignments match as follows (see also figure 4)

$$
\sigma\left(\begin{array}{lllllll}
\hat{j}_{1} & \hat{j}_{1}^{*} & \hat{j}_{2} & \hat{j}_{2}^{*} & \ldots & \hat{j}_{2 n} & \hat{j}_{2 n}^{*} \\
\hat{l}_{1} & \hat{l}_{1}^{*} & \hat{l}_{2} & \hat{l}_{2}^{*} & \ldots & \hat{l}_{2 n} & \hat{l}_{2 n}^{*}
\end{array}\right) .
$$




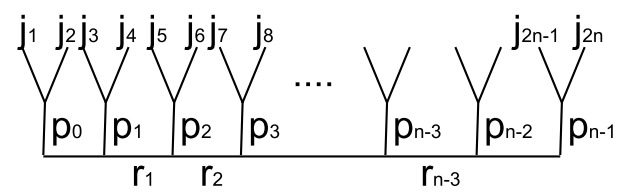

Figure 5: The conformal block of type $\{\mathbf{p} ; \mathbf{r}\}$ (odd).

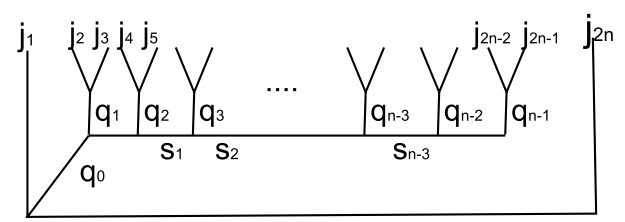

Figure 6: The conformal block of type $\{\mathbf{q} ; \mathbf{s}\}$ (even).

Since any (colored oriented) link can be obtained as the plat closure of a braid [64], we do not lose generality for what concerns the class of links that can be handled.

The further step consists in embedding the $2 n$-strand braid into a threesphere $S^{3}$ with two three-balls removed, giving rise to a three-manifold with two boundaries $\Sigma^{1}, \Sigma^{2}$ (topologically 2 two-spheres $S^{2}$ with opposite orientations). The intersections ("punctures") of the braid (2.11) with the boundaries inherit the colorings and orientations from the corresponding strands of the braid (to be associated with Wilson line operators in the ambient CSW topological field theory). Following [8], finite-dimensional Hilbert spaces $\mathcal{H}^{1} \otimes \mathcal{H}^{2}$ are associated with to the two boundaries $\Sigma^{1}, \Sigma^{2}$ and the basis sets in these spaces are the so-called conformal blocks of the boundary WZW conformal field theory at level $\ell$, with $2 n$ external lines labelled by (different) irreps of $\mathrm{SU}(2)_{q}$ ( $\ell$ is related to CSW coupling constant by $\ell=k+2$, so that from now on we set $q=\exp \{2 \pi \mathrm{i} / \ell\})$. Two particular types of conformal block bases are needed to deal with braids the plat closures of which will give rise to colored oriented links, and their combinatorial patterns are shown in figures 5 and 6 .

The (orthonormal) basis sets are constructed by taking particular binary coupling schemes of the $2 n$ "incoming" angular momentum variables $j_{1}$, $j_{2}, \ldots, j_{2 n}$ which must sum up to give a spin-0 total singlet state. ${ }^{3}$ The procedure can be carried out by parallelling the $\mathrm{SU}(2)$, undeformed case (see Appendix A) but here the labels of the irreps $j_{i}$ 's (integer and half-integers) are constrained to range from 0 to $\ell / 2$ and the binary bracketings on tensor

\footnotetext{
${ }^{3}$ Incidentally, such a choice complies also with the conditions necessary for the construction of error avoiding codes and implies as well the robustness of the scheme $[65,66]$.
} 
products are decomposed according to rules of $\mathrm{SU}(2)_{q}$ representation theory (see, e.g., [61, Section 3]).

Looking at the combinatorial structure of figure 5, the most general oddcoupled basis is consistently labelled as

$$
|\mathbf{p} ; \mathbf{r}\rangle^{\mathbf{j}}
$$

where $\mathbf{j}$ stands for the ordered string $j_{1}, j_{2}, \ldots j_{2 n}, \mathbf{p} \equiv p_{0}, p_{1}, \ldots, p_{n-1}$ and $\mathbf{r} \equiv r_{1}, r_{2}, \ldots, r_{n-3}$. In the even-coupled case depicted in figure 6 , the states of the basis are denoted by

$$
|\mathbf{q} ; \mathbf{s}\rangle \mathbf{j}
$$

where $\mathbf{j}$ is the same as before while $\mathbf{q} \equiv q_{0}, q_{1}, \ldots, q_{n-1}$ and $\mathbf{s} \equiv s_{1}, s_{2}, \ldots$, $s_{n-3}$.

The basis vectors associated to the conformal blocks (2.12) and (2.13) are related to each other by

$$
|\mathbf{p} ; \mathbf{r}\rangle^{\mathbf{j}}=\sum_{(\mathbf{q} ; \mathbf{s})} A_{(\mathbf{p} ; \mathbf{r})}^{(\mathbf{q} ; \mathbf{r})}\left[\begin{array}{cc}
j_{1} & j_{2} \\
j_{3} & j_{4} \\
\vdots & \vdots \\
j_{2 n-1} & j_{2 n}
\end{array}\right]|\mathbf{q} ; \mathbf{s}\rangle^{\mathbf{j}}
$$

where the symbol $A_{(\mathbf{p} ; \mathbf{r})}^{(\mathbf{q} ; \mathbf{s})}[::]$ represents the unitary duality matrix (or $q$-deformed $3 n j$ recoupling coefficient). As pointed out in Section 2.1, it is a standard result that any such duality matrix can be decomposed into (sums of) products of "basic" duality matrices or $q-6 j$ symbols, see Section 3.2 below.

A graphical representation of the decomposition (2.14) in the case of eight incoming spin variables is shown in figure 7 . Note that the graphical representation of the basic duality transformation (the matrix counterpart of the associator in the language of tensor categories) can be also drawn in the most familiar form shown in figure 8.

As proved in [28], the colored polynomial of a link $L$, presented as the plat closure $\hat{\sigma}$ of a colored braid $\sigma\left(\begin{array}{lllll}\hat{j}_{1} & \hat{j}_{1}^{*} & \ldots & \hat{j}_{n} & \hat{j}_{n}^{*} \\ \hat{l}_{1} & \hat{l}_{1}^{*} & \ldots & \hat{l}_{n} & \hat{l}_{n}^{*}\end{array}\right)$ as defined in (2.11), is given by

$$
J[L ; \mathbf{j} ; q]=\operatorname{Tr} \prod_{i=1}^{n}\left[2 j_{i}+1\right]_{q}^{\mathbf{l}}\left\langle\mathbf{0} ; \mathbf{0}\left|\hat{U}\left[\sigma\left(\begin{array}{ccccc}
\hat{j}_{1} & \hat{j}_{1}^{*} & \ldots & \hat{j}_{n} & \hat{j}_{n}^{*} \\
\hat{l}_{1} & \hat{l}_{1}^{*} & \ldots & \hat{l}_{n} & \hat{l}_{n}^{*}
\end{array}\right)\right]\right| \mathbf{0} ; \mathbf{0}\right\rangle^{\mathbf{j}}
$$




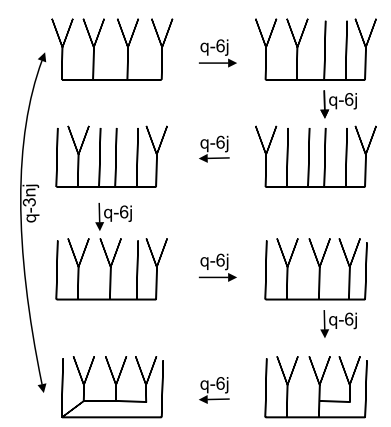

Figure 7: Example of decomposition of a duality transformation between the two extremal conformal blocks in the case of eight incoming spins.

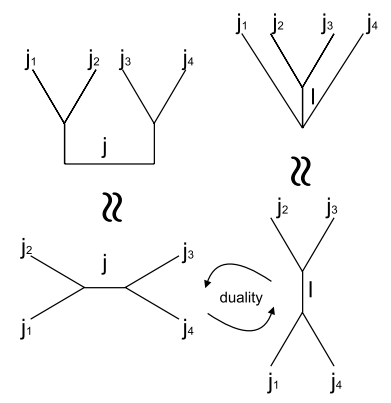

Figure 8: The elementary duality transformation associated with a $q-6 j$ symbol.

where $\mathbf{j} \equiv\left(j_{1}, j_{2}, \ldots, j_{2 n}\right),\left[2 j_{i}+1\right]_{q}$ is the quantum dimension of the irrep labelled by $j_{i}$ and the quantum integer $[x]_{q}$ is defined as

$$
[x]_{q} \equiv \frac{x^{q / 2}-x^{-q / 2}}{x^{1 / 2}-x^{-1 / 2}} .
$$

Thus $J[L ; \mathbf{j} ; q]$ can be evaluated by taking the trace of the matrix elements of the composite braiding operator $\hat{U}[\sigma]$ in the Kaul representation with respect to the odd-coupled basis, where all the intermediate quantum numbers are constrained to give singlet eigenstates (a similar result would hold true for the even-coupled basis). Moreover, $\hat{U}[\sigma]$ can be decomposed into a finite sequence of unitary matrices $U\left[\sigma_{2 l+1}\right]$ (diagonal matrices in the oddcoupled basis adopted in (2.15)) and duality matrices of the type (2.14) to be applied whenever a switch to the even-coupled basis is needed, namely when an even $U\left[\sigma_{2 l}\right]$ occurs in the decomposition (see [28] for the explicit expression of these matrices). 
The construction outlined above can be cast into an effective process of calculation by resorting the concept of quantum recognizer introduced in Section 2.1.

The $\mathcal{A}_{q}$ recognizer is defined, for a fixed root of unity $q=\exp \{2 \pi i / \ell\}$, by the five-tuple $\left\{\mathcal{C}_{\text {odd }}, \mathcal{H}, B_{2 n}\right.$, $\{$ accept, reject, $\left.\epsilon\}, U\left(B_{2 n}\right)\right\}$, where

- $\mathcal{C}_{\text {odd }}$ is the odd-coupled conformal block basis of the boundary WZW theory (see (2.12) and figure 5).

- $\mathcal{H}$ is the ordered tensor product of $2 n\left(2 j_{i}+1\right)$-dimensional Hilbert spaces supporting irreps of $\mathrm{SU}(2)_{q}$ labelled by $j_{i}$, with $j_{i} \leq(\ell-2) / 2$ $(i=1,2, \ldots, 2 n)$.

- $B_{2 n}$ is the braid group on $2 n$ strands whose generators $g \equiv\left\{\sigma_{1}, \sigma_{2}, \ldots\right.$, $\left.\sigma_{2 n-1}\right\}$ and their inverses represent the input alphabet (we may add the identity element $e \in B_{2 n}$ as null symbol).

- $Y=\{$ accept, reject, $\epsilon\}$ is the output alphabet.

- The transition matrices are expressed in terms of $U\left(B_{2 n}\right)$, denoting collectively the Kaul unitary representation matrices, while the projectors $P(y)(y \in Y)$ are defined as in the general case given in Section 2.1.

According to the above definitions, we provide the automaton with an input word $w \in B_{2 n}$ of length $\kappa$ (written in the alphabeth $g$ by natural composition in $B_{2 n}$ )

$$
w=\sigma_{\alpha_{1}}^{\epsilon_{1}} \sigma_{\alpha_{2}}^{\epsilon_{2}} \cdots \sigma_{\alpha_{\kappa}}^{\epsilon_{\kappa}} ; \quad \sigma_{\alpha_{i}} \in g, \epsilon_{i}= \pm 1
$$

and such that the (plat) closure $\hat{w}$ of the $2 n$-strand braid $w$ gives the link $L$ to be processed. Dropping for simplicity all the matrix indices, the unitary evolution of the automaton is achieved by applying the sequence

$$
U(w)=U\left(\sigma_{\alpha_{\kappa}}^{\epsilon_{\kappa}}\right) U\left(\sigma_{\alpha_{\kappa-1}}^{\epsilon_{\kappa-1}}\right) \cdots U\left(\sigma_{\alpha_{1}}^{\epsilon_{1}}\right)
$$

to a start ket $|\mathbf{0} ; \mathbf{0}\rangle \mathbf{j}^{\mathbf{j}}$ in the odd-coupled basis (2.12).

Whenever an odd-braiding $\sigma_{\alpha}=\sigma_{2 i+1}\left(\right.$ or $\left.\left(\sigma_{2 i+1}\right)^{-1}\right)$ occurs, the automaton one-step evolution upgrades the internal state by inserting the eigenvalue of the associated unitary $U$. On the other hand, when an even-braiding $\sigma_{\beta}=\sigma_{2 i}\left(\right.$ or $\left.\left(\sigma_{2 i}\right)^{-1}\right)$ must be implemented, the automaton has to change the parity of the internal state by means of a duality matrix (2.14), so that the effective transformation is given by the product $U\left(\sigma_{\beta}\right) A[::]$. Since any duality transformation can be split into a sequence of basic duality matrices, we may look at the $q-6 j$ symbol as representing an "elementary", one-step evolution of the automaton. By resorting to standard results in graph theory it is possible to estimate how many $q-6 j$ symbols are needed to decompose the 
most general $q$-recoupling coefficient $[2,67]$. In the present case the upper bound can be expressed in terms of $2 n$, the braid index (or, equivalently, the number of strands of the input braid). On the basis of the above remarks, the time complexity function (number of computational steps) for processing a braid-word of length $\kappa$ on the quantum recognizer $\mathcal{A}_{q}$ is bounded from above by [2]

$$
\kappa(\tilde{N} \ln \tilde{N}) \quad \text { where } \tilde{N} \equiv(2 n-1),
$$

implying that the automaton processes efficiently such braids.

Let us finally comment on the "probability distribution" entering into the definition of a quantum automaton that recognizes a language in a probabilistic sense (end of Section 2.1). On the basis of the expression of the colored link invariant given in (2.15) and by comparison with the word probability of a quantum recognizer defined in (2.7), it should be quite clear that the probability naturally associated with a link $L$ processed on $\mathcal{A}_{q}$ is the square modulus of its colored polynomial (note that the positivity conditions required in (2.5) are always satisfied).

In order to check this result in a concrete case, the explicit construction of the $q$-spin network automaton that recognizes the braid group language with a probability distribution given by the square modulus of the Jones polynomial is carried out in Appendix B.

\section{Efficient quantum algorithms for three-manifold quantum invariants}

\subsection{Colored framed links and three-manifold quantum invariants}

The quantum invariants of three-manifolds that we are going to discuss within the mathematical framework developed in [30], see also [68] — can be obtained as combinations of polynomial invariants of "framed" unoriented links in the three-sphere $S^{3}$ on the basis of theorem 3.1 stated below. It is worth noting that in the CSW environment the necessity of introducing framings is physically motivated by the requirement of general covariance of the quantized field theory (see, e.g., [23, Chapter 3]).

Loosely speaking, a framed oriented link $[L ; \mathbf{f}]$ is obtained from a link $L$ - thought of as made of knotted strings - by thickening its strings to get oriented "ribbons". If $L$ has $S$ knot components $K_{1}, K_{2}, \ldots, K_{S}$, for each $K_{s}$ we introduce another closed path $K_{s}^{f}$ oriented in the same way as 
$K_{s}$ and lying within an infinitesimal neighborhood of $K_{s}$ (knots and links are embedded in $\mathbb{R}^{3}$ or in $S^{3}$ ). The overall topology of the link is not modified, but for each $K_{s}$ we now have an extra variable $\tau\left(K_{s}\right)$ telling us how many times the oriented ribbon is "twisted". Denoting by $\mathbf{f} \doteq\left\{f_{s}=n(s), n(s) \in\right.$ $\mathbb{Z}\}(s=1,2, \ldots, S)$ the framing of the link $L, f_{s}$ is the self-linking number of the band, or equivalently the linking number $l k\left(K_{s}, K_{s}^{f}\right) \equiv \chi\left(K_{s}, K_{s}^{f}\right)$ between the knot $K_{s}$ and its framing curve $K_{s}^{f}$ which winds $n(s)$ times in the right-handed direction. The twist of the band $\tau\left(K_{s}\right)$ is not independent from $l k\left(K_{s}, K_{s}^{f}\right)$, and the simplest choice we can made is to set

$$
\tau\left(K_{s}\right)=w\left(K_{s}\right)
$$

where $w\left(K_{s}\right)$ is the writhe of the $s$ th component. ${ }^{4}$

The type of framing usually adopted is the "vertical" framing, where the frame is thought to be placed vertically above the link diagram. Looking at figure 9 , the framing can be represented by putting the writhe number $w\left(K_{s}\right) \equiv n(s)$ nearby the band or even by writing $n(s)$ next to the string representing (a portion of) $K_{s}$.

The three-manifolds we are going to consider are closed, connected and oriented, namely compact and without boundary. In general any such manifold $\mathcal{M}^{3}$ can be presented as the union of several components - endowed with two-dimensional boundaries - glued together by suitable identification prescriptions on the points lying in their boundaries. If the components are sewed together in a different way a topologically different manifold $\widetilde{\mathcal{M}^{3}}$ may be obtained (recall that two manifolds are topologically equivalent iff they are homeomorphic, namely there exists a one-to-one continuous map

\footnotetext{
${ }^{4}$ Given a link diagram $D(L)$, namely a projection of the oriented link $L$ onto a plane, we can define two numerical invariants associated with such diagrams. The writhe number $w\left(D_{L}\right)$ is given by $w\left(D_{L}\right)=\sum_{p} \epsilon(p)$ where $\{p\}$ are the crossing points $\in D(L)$ and $\epsilon(p)= \pm 1$ according to whether there appears an over-crossing of the left strand over the right strand or an under-crossing (both strands are oriented upward). The linking number, defined for a link with more than one component knot, is defined for each pair of components $\left(K_{i}, K_{j}\right)$ as $l k\left(K_{i}, K_{j}\right)=w\left(D_{L}\right)-w\left(D_{K_{i}}\right)-w\left(D_{K_{j}}\right)$.

It can be shown that the writhe is a regular isotopy invariant for knots and link and the linking number is an ambient isotopy invariant for links. Recall that two links in $\mathbb{R}^{3}$ (or $S^{3}$ ) are ambient isotopic if they can be continuously deformed one into the other. It can be shown that two links are ambient isotopic if and only if their diagrams are connected by a finite sequence of Reidemeister moves of type I, II, II. Regular isotopy is a restricted type of equivalence among links where the allowed Reidemeister moves are of type II and III. It is worth noting that the colored link polynomials (2.15) are invariants of regular isotopy while an associated ambient isotopy invariant can be obtained by multiplying $J(L ; \mathbf{j} ; q)$ by $\left\{q^{-3 w(L) / 4} /\left(q^{1 / 2}-q^{-1 / 2}\right)\right\}$, where $w(L)$ is the writhe defined above.
} 


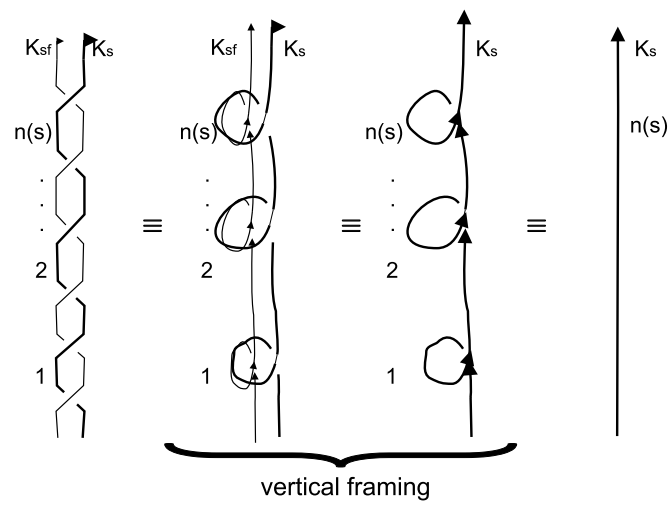

Figure 9: Graphical representations for the vertical framing.

between them). The new manifold $\widetilde{\mathcal{M}^{3}}$ is said to be obtained from $\mathcal{M}^{3}$ by means of "surgery."

The fundamental theorem that characterizes (equivalence classes of homeomorphic) three-manifolds reads $[69,70]$

Theorem 3.1. Every closed, connected and orientable three-manifold $\mathcal{M}^{3}$ can be obtained by surgery on an unoriented framed link in the three-sphere $S^{3}$.

Following [23], let us illustrate in some details the (Dehn) surgery procedure referred to in the statement of the theorem in the simple case of a knot $K$ (one-component link) $\subset S^{3}$. The building blocks of the construction are solid tori, so let us begin the discussion by considering the two-dimensional torus $\mathcal{T}^{2}=S^{1} \times S^{1}$ (the cartesian product of two unit circles). A point in $\mathcal{T}^{2}$ can be specified by the coordinates $\left(\mathrm{e}^{\mathrm{i} \theta_{1}}, \mathrm{e}^{\mathrm{i} \theta_{2}}\right)$ in the complex plane and in particular a point lying on the longitude has coordinate $\left(\mathrm{e}^{\mathrm{i} \theta_{1}}, 1\right)$ while a point in a meridian has coordinate $\left(1, \mathrm{e}^{\mathrm{i} \theta_{2}}\right)$. A solid torus $\mathcal{V}^{3}$ is a threemanifold homeomorphic to $S^{1} \times \mathcal{D}^{2}$, where $\mathcal{D}^{2}$ is the two-dimensional disc. The boundary $\partial \mathcal{V}^{3}$ of $\mathcal{V}^{3}$ is the torus $\mathcal{T}^{2}$.

A framing on the solid torus is a particular homeomorphism $h: S^{1} \times$ $\mathcal{D}^{2} \rightarrow \mathcal{V}^{3}$. Given a framing $h$ of $\mathcal{V}^{3}, h\left(1 \times \partial \mathcal{D}^{2}\right)$ is a meridian while $h\left(S^{1} \times 1\right)$ is a longitude.

A tubular neighborhood $\mathcal{N}$ of a knot $K$ in $S^{3}$ is an embedding $\iota: K \times \mathcal{D}^{2} \rightarrow$ $S^{3}$ such that $\iota(x, 0)=x \forall x \in K$. The framing $K^{f}$ of a knot $K$ is defined as the framing of the tubular neighborhood of the knot. In particular, the standard framing $K^{f}$ of a knot $K$ is such that the linking number $\chi\left(K, K^{f}\right)$ is equal to zero. 
Representing the unit disc $\mathcal{D}^{2}$ in the complex plane, the points of $S^{1} \times \mathcal{D}^{2}$ have coordinates $\left(\mathrm{e}^{\mathrm{i} \theta_{1}}, r \mathrm{e}^{\mathrm{i} \theta_{2}}\right)$ with $0 \leq r \leq 1$. The self-homeomorphisms $\hat{t}$ of $S^{1} \times \mathcal{D}^{2}$, defined explicitly by

$$
\hat{t}_{ \pm}\left(\mathrm{e}^{\mathrm{i} \theta_{1}}, r \mathrm{e}^{\mathrm{i} \theta_{2}}\right)=\left(\mathrm{e}^{\mathrm{i} \theta_{1}}, r \mathrm{e}^{\left(\theta_{2} \pm \theta_{1}\right)}\right),
$$

are the basic twist operations of $S^{1} \times \mathcal{D}^{2}$.

Given a tubular neighborhood $\mathcal{N}$ of a knot $\mathcal{K}$ with standard framing $f$ : $S^{1} \times \mathcal{D}^{2} \rightarrow \mathcal{N}$, the right-handed and left-handed twist $t_{ \pm}$of $\mathcal{N}$ are related to the $\hat{t}_{ \pm}$by

$$
t_{ \pm}=f \hat{t}_{ \pm} f^{-1} .
$$

A Dehn surgery performed along a (framed) knot $K \subset S^{3}$ can be described as follows:

1. remove the interior $\stackrel{\circ}{\mathcal{N}}$ of a tubular neighborhood $\mathcal{N}$ of $K$ (the resulting manifold $\left(S^{3} \backslash \mathcal{N}\right)$ is the complement torus);

2. consider $\left(S^{3} \backslash \stackrel{\circ}{\mathcal{N}}\right)$ and $\mathcal{N}$ as distinct spaces;

3. glue back $\mathcal{N}$ and $\left(S^{3} \backslash \stackrel{\circ}{\mathcal{N}}\right)$ by identifying the points in their boundaries through a given homomorphism $h: \partial \mathcal{N} \rightarrow \partial\left(S^{3} \backslash \stackrel{\circ}{\mathcal{N}}\right)$.

The resulting manifold $\mathcal{M}_{K}^{3}$ is recovered by setting

$$
\mathcal{M}_{K}^{3}=\left(S^{3} \backslash \stackrel{\circ}{\mathcal{N}}\right) \bigcup_{h} \mathcal{N}
$$

and it is completely specified by the knot $K$ and by the choice of the gluing homomorphism $h$. Equivalently, the surgery is characterized by the knot $K$ and by a closed curve $\gamma \in \partial \mathcal{N}$ representing $h(\mu)$, where $\mu$ is the meridian of $\mathcal{N}$.

Dehn surgery is a simple and constructive prescription which basically consists in removing and sewing back solid tori from the three-sphere. However, since different surgery instructions may give rise to homeomorphic manifolds, it is crucial to define the equivalence relations that identify the surgery instructions providing the same (homeomorphism class of) threemanifold. Once these rules are taken into account, the classification problem for three-manifolds can be actually reduced to the problem of classification of knots (links).

The equivalence relations among surgery instructions yielding a same three-manifold are topological operations on framed link diagrams known as Kirby moves. 


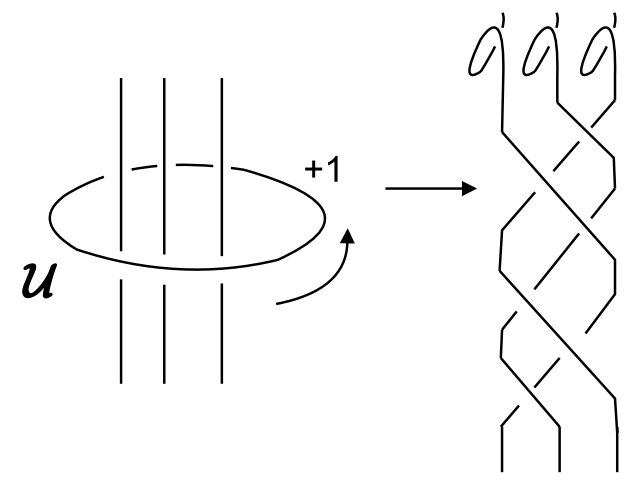

Figure 10: The first Kirby move.
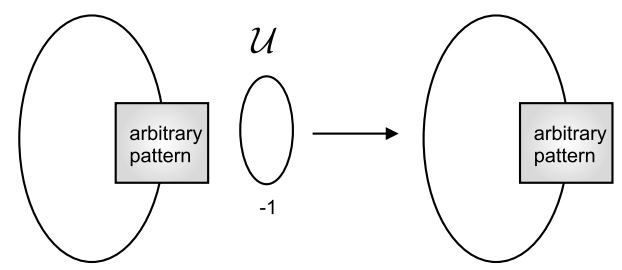

Figure 11: The second Kirby move.

I move. The configuration described by the unknot $\mathcal{U}$, with framing +1 , enclosing $n$ unlinked strands $K_{i}$, with framing $f_{i}$, lying on a ribbon can be changed into the configuration where $\mathcal{U}$ is removed and the ribbon is twisted in the clockwise direction from below, see figure 10. The framing of the components $K_{i}$ 's must be changed according to

$$
f_{i} \mapsto f_{i}^{\prime}=f_{i}-\chi^{2}\left(K_{i}, \mathcal{U}\right),
$$

where $\chi^{2}\left(K_{i}, \mathcal{U}\right)$ is the square of the linking number between $K_{i}$ and $\mathcal{U}$.

II move. An unknotted link $\mathcal{U}$ with framing -1 can be removed without affecting the rest of the link, see figure 11.

III move. This is the "inverse" of the first move and amounts to change the configuration containing $\mathcal{U}$ with framing -1 into the anti-clockwise twisted ribbon of $K_{i}$ 's and without $\mathcal{U}$. Here the framings change according to

$$
f_{i} \longmapsto f_{i}^{\prime}=f_{i}+\chi^{2}\left(K_{i}, \mathcal{U}\right) .
$$

IV move. This is the "inverse" of the second move. An unknotted link $\mathcal{U}$ with framing +1 can be removed without affecting the rest of the link. 
The extension of the results stated above in order to deal with $\mathrm{SU}(2)_{q^{-}}$ colored surgery links to be associated with three-manifold invariants can be carried in a quite straightforward way. The construction developed in $[2,3]$ and summarized in Section 2.3 basically relies on Kaul unitary representation of (colored) oriented braids on $2 n$ strands [28] and thus we need preliminarily to generalize such a representation to deal with unoriented braids and links. The goal can be achieved by suitably modifying the eigenvalues of the elementary braiding matrices in the odd-coupled basis $|\mathbf{p} ; \mathbf{r}\rangle \mathbf{j}$ introduced in (2.12) according to [29]

$$
\begin{aligned}
& U\left(\sigma_{2 l+1}\right)|\mathbf{p} ; \mathbf{r}\rangle^{\left(\ldots, j_{2 l+1}, j_{2 l+2}, \ldots\right)}=\lambda_{l}|\mathbf{p} ; \mathbf{r}\rangle^{\left(\ldots, j_{2 l+1}, j_{2 l+2}, \ldots\right)} \quad \text { with } \\
& \lambda_{l} \doteq \lambda\left(j_{2 l+1}, j_{2 l+2}\right) \equiv \lambda_{l}\left(j, j^{\prime}\right)=(-)^{\left|j-j^{\prime}\right|-l} q^{ \pm\left(c_{j}+c_{j^{\prime}}-c_{l}\right) / 2}
\end{aligned}
$$

where $c_{j}=j(j+1)$ and $c_{j^{\prime}}=j^{\prime}\left(j^{\prime}+1\right)$ are the quadratic Casimir invariants associated with the irreps $j, j^{\prime}$. The \pm 1 in the exponent of the parameter $q$ refer to left-handed (respectively, right-handed) half-twists in two parallel strands carrying the coloring $j, j^{\prime}$ and it can be easily checked that these eigenvalues do not depend on the orientations but only on the over/ under-crossing features (the colored strands are ordered from left to right as happened for oriented braids, see (2.9) and figure 3).

It is worth noting that the duality matrices (2.14) — needed whenever an even braiding $U\left(\sigma_{2 l}\right)$ has to be applied - are independent from orientations of the strands, so that they can be used in the present context with no further modification.

According to Theorem 3.1, what we really have to handle are colored links in the vertical framing $\mathbf{f}$ and then the effect of adding or deleting a \pm 1 in the writhe of the link must be properly taken into account (this operation in the standard framing would not affect the topology of the link). Referring in particular to a $j$-colored framed unknot $\mathcal{U}$, the associated link invariant turns out to be changed into

$$
\begin{aligned}
& J[\mathcal{U} ; j,+1 ; q]=q^{c_{j}} J[\mathcal{U} ; j, 0 ; q], \\
& J[\mathcal{U} ; j,-1 ; q]=q^{-c_{j}} J[\mathcal{U} ; j, 0 ; q],
\end{aligned}
$$

where \pm 1 on the left-handed sides denote the \pm 1 vertical framing whereas in the right-handed sides there appears the knot invariant of the unknot in the standard 0 -framing, whose numerical value is given by the $q$-dimension $[2 j+1]_{q}$ defined in $(2.16)$. The latter relations provide in practice the operatorial content of Kirby moves applied to framed colored links. Then the 
requirement of invariance under Kirby moves of the forthcoming threemanifold topological invariants makes it necessary to compensate the effects of (3.7) by resorting to properties of the so-called linking matrix.

For a framed link $[L ; \mathbf{f}]$ whose components $K_{1}, K_{2}, \ldots, K_{S}$ have framings $n_{1}, n_{2}, \ldots, n_{S}$ respectively, the linking matrix is a symmetric matrix defined as

$$
\chi[L ; \mathbf{f}]=\left(\begin{array}{ccccc}
n_{1} & \chi\left(K_{1}, K_{2}\right) & \chi\left(K_{1}, K_{3}\right) & \cdots & \chi\left(K_{1}, K_{S}\right) \\
\chi\left(K_{2}, K_{1}\right) & n_{2} & \chi\left(K_{2}, K_{3}\right) & \cdots & \chi\left(K_{2}, K_{S}\right) \\
\vdots & \cdots & \cdots & \cdots & \vdots \\
\chi\left(K_{S}, K_{1}\right) & \cdots & \cdots & \cdots & n_{S}
\end{array}\right)
$$

where $\chi\left(K_{i}, K_{j}\right)$ is the linking number between the component knots $K_{i}$ and $K_{j}$. The signature of the linking matrix, denoted by $\sigma[L ; \mathbf{f}]$, is the difference between the number of positive and negative eigenvalues of $\chi[L ; \mathbf{f}]$.

With these preliminary definitions, let us state the following theorem, the original proof of which can be found in $[12,30]$ (see also [68]).

Theorem 3.2. For a closed, connected and oriented three-manifold $\mathcal{M}_{L}^{3}$ obtained by surgery in the three-sphere along an unoriented colored framed link $[L ; \mathbf{f}, \mathbf{j}]$ with $S$ link components and for any fixed root of unity $q=\mathrm{e}^{\frac{2 \pi \mathrm{i}}{k+2}}$ the quantity

$$
\mathcal{I}\left[\mathcal{M}_{L}^{3} ; \mathbf{f} ; q\right]=\alpha^{-\sigma[L ; \mathbf{f}]} \sum_{\{\mathbf{j}\}} \mu_{j_{1}} \mu_{j_{2}} \cdots \mu_{j_{S}} J[L ; \mathbf{f}, \mathbf{j} ; q]
$$

is a topological invariant of the three-manifold endowed with the framing assignment $\mathbf{f} .^{5}$

Here $\alpha \equiv \exp \frac{3 \pi i k}{4(k+2)}, \quad \mu_{j}=\sqrt{\frac{2}{k+2}} \sin \frac{\pi(2 j+1)}{k+2}, \quad \mathbf{j} \equiv\left(j_{1}, j_{2}, \ldots, j_{S}\right)$ run over $\left\{0, \frac{1}{2}, \ldots, \frac{k}{2}\right\}$ and the summation is performed over all admissible colorings.

\footnotetext{
${ }^{5}$ This means in practice that, on applying Kirby moves I-IV to $[L ; \mathbf{f}] \subset S^{3}$, the value of the invariant does not change, namely depends only on the homeomorphism class of the three-manifold. Note however that we are not in the presence of a complete three-manifold invariant since there exist topologically distinct manifolds with the same $\mathcal{I}$.

The extension of the theorem to deal with surgery operations performed on manifolds topologically different from $S^{3}$ and to situations in which $q$-deformations of other semisimple Lie groups are involved can be found in the reference quoted above. All such invariants are collectively referred to as three-manifold "quantum invariants" or even as "state sum models" by noticing that an expression like (3.9) can be interpreted as a partition function over suitably weighted "states" represented by the link polynomials with different colorings.
} 
$J[L ; \mathbf{f}, \mathbf{j} ; q]$ in the previous expression is the "unoriented" counterpart of the polynomial for the link $L$ with coloring assignment $\mathbf{j}$ on its components and the summation is performed over all admissible colorings.

It is worth noting that the presentation of the colored links used both in Section 2.2 (see (2.15)) and in (3.6) is slightly different from the presentation given in Theorem 3.2 above, even though we keep on using the same notation $\mathbf{j}$ for the colorings. In the latter case a coloring is assigned to each of the $S$ link components, whereas in the former we label the $2 n$ strands of the associated braid with $n$ colors (see, e.g., figure 4).

However, what really matters is the fact that both presentations of the link $L$ give rise to the same invariant, as could be proved by exploiting the properties of the $\mathrm{SU}(2)_{q}-$ representation ring. From the computational viewpoint this twofold choice does not matter as well because we could efficiently implement the transformation from a given link diagram to any associated closed braid on a classical computer (see, e.g., Section 2 of [61] for a discussion on classical algorithmic questions about braids and links). Expression (3.9) makes it manifest the overall dependence on the framing in the factor $\alpha^{-\sigma[L ; \mathbf{f}]}$, but in what follows we are going to express the link polynomials $J[L ; \mathbf{f}, \mathbf{j} ; q]$ as expectation values of Kaul unitary representation [29] worked out for an unoriented $2 n$-strands braid the plat closure of which gives the link under examination.

\subsection{Quantum algorithm for approximating 3-manifold invariants}

In this section we shall describe the quantum algorithm for computing the colored polynomial $J[L ; \mathbf{f}, \mathbf{j} ; q]$ and the associate invariant $\mathcal{I}\left[\mathcal{M}_{L}^{3} ; \mathbf{f} ; q\right]$ defined in (3.9). This algorithm is an extension of the quantum algorithm proposed in $[2,3]$ which efficiently approximated the value of the colored Jones polynomials (2.15).

As anticipated in Section 1.2 we need in the present context the notion of additive approximation introduced [14] (see also [16]). Given a normalized function $g(x)$, where $x$ denotes an instance of the problem, we have an additive approximation of its value for each $x$ if we can associate with $g(x)$ a random variable $Z$ such that

$$
\operatorname{pr}\{|g(x)-Z| \leq \eta\} \geq 3 / 4,
$$

for any $\eta \geq 0$. Moreover, the time needed to achieve the approximation must be polynomial in the size of the problem and in $\eta^{-1}$. Then the problem we are interested in can be stated formally as follows. 


\subsubsection{Approximating three-manifold invariants}

Given a framed link $[L ; \mathbf{f}]$ with component knots $K_{1}, K_{2}, \ldots, K_{S}$, framing $\mathbf{f}=\left(n_{1}, n_{2}, \ldots, n_{S}\right)$, a positive integer $k$, and a set of allowed colors $\mathbf{j}=$ $\{0,1 / 2, \ldots, k / 2\}$, we want to sample out a random variable $Z$ representing an additive approximation of the value of the normalized threemanifold invariant $\mathcal{I}\left[\mathcal{M}_{L}^{3} ; \mathbf{f} ; q\right]$ evaluated at $q=\mathrm{e}^{\frac{2 \pi \mathrm{i}}{k+2}}$ - in such a way that the following condition holds true:

$$
\operatorname{pr}\left\{\left|\mathcal{I}\left[\mathcal{M}_{L}^{3} ; \mathbf{f} ; q\right]-Z\right| \leq \eta\right\} \geq 3 / 4
$$

The size of the problem is expressed in terms of the number of crossings $\kappa$ of the surgery link $L$ and by the number of strands of the associate braid, (as it happened for colored link polynomials), but we shall need to handle properly the whole set of allowed colorings $\mathbf{j}$ and the framing $\mathbf{f}$ as well. ${ }^{6}$

As anticipated in Section 2.1, the quantum algorithms for evaluating (additive approximations of) topological invariants in the framework of quantum CSW theory are based on a two-level procedure outlined already in [3] in connection with colored link polynomials and improved here for threemanifold invariants. The rationale underlying our procedure is briefly summarized below while technical details are developed in the rest of the section.

(A) Within the computational model of the $q$-deformed spin network recognizer $\mathcal{A}_{q}$ (Section 2) both topological and field-theoretic data encoded into a framed colored link $[L ; \mathbf{f}, \mathbf{j}]$ associated with a unitary braiding operator in the Kaul representation [29] — are efficiently processed. The estimate of the time complexity function (number of computational steps) required to complete the calculation given in (2.19) still holds true.

(B) By resorting to standard quantum circuit model techniques and related approximation schemes it can be shown that

(i) the start state $|\psi\rangle^{\mathcal{I}}$ of the recognizer $\mathcal{A}_{q}$ needed for processing the three-manifold invariant $\mathcal{I}\left[\mathcal{M}_{L}^{3} ; \mathbf{f} ; q\right]$ is efficiently encoded into a qubit register;

(ii) the braiding operator associated with the framed link $[L ; \mathbf{f}, \mathbf{j}]$ already split into a sequence of "elementary" braiding and duality transformations on the basis of the recognizer design — can be efficiently compiled on a standard quantum circuit applied to the start qubit register;

\footnotetext{
${ }^{6}$ From now on we agree that the invariant is normalized by the product of the $q$-dimensions associated with the link components, namely by the factor $\prod_{i=1}^{S}\left[2 j_{i}+1\right]_{q}$.
} 
(iii) by resorting to the Hadamard test [15] — an efficient sampling procedure which provides the expectation value of an unitary operator on a (qubit) state - it is possible to estimate the value of the invariant $\mathcal{I}\left[\mathcal{M}_{L}^{3} ; \mathbf{f} ; q\right]$ from a series of measurements on an ancilla qubit coupled to the start state.

This two-level computational process for the approximation of three-manifold invariants in the sense of (3.11) is thus efficient with respect to the standard model of quantum computation and not simply from the viewpoint of the $q$-deformed automaton model.

(C) The expectation value of the braiding operator evaluated on the start state and sampled as described above, can in turn be related to a suitable probability distribution on the language(s) recognized by $\mathcal{A}_{q}$. In view of the properties of such distributions (cf. the concluding remarks of Sections 2.1 and 2.2 and Appendix B), the whole procedure can be reinterpreted by saying that the automaton recognizes the language of the braid group with a probability distribution given by the square modulus of the (normalized) invariant $\mathcal{I}\left[\mathcal{M}_{L}^{3} ; \mathbf{f} ; q\right]$.

Given an unoriented link $L$ presented as the plat closure of a $2 n$-strands braid with a fixed set of colorings $\mathbf{j} \equiv j_{1}, j_{2}, \ldots, j_{2 n}$ (see Section 2.2 for the oriented case, in particular figure 4), let us denote again the conformal block odd-coupled basis of the boundary WZW theory by $|\mathbf{p} ; \mathbf{r}\rangle^{\mathbf{j}}$ as in (2.12) and figure 5. Here $\mathbf{p}=p_{0}, \ldots, p_{n-1}$ and $\mathbf{r}=r_{0}, \ldots, r_{n-3}$ and both $\mathbf{j}$ and the intermediate quantum numbers $\mathbf{p}, \mathbf{r}$ take values in the collection $\{0,1 / 2, \ldots, k / 2\}$, bounded form above by the coupling constant $k$ of quantum CSW theory.

Each spin quantum number can be encoded into a qubit register made of $\left\lceil\log _{2}(k+1)\right\rceil$ qubits, where $\lceil x\rceil$ denotes the smallest integer $\geq x$, see figure 12 .

Since an element of the basis is specified by $(4 n-3)$ quantum numbers $(\mathbf{j}, \mathbf{p}$ and $\mathbf{r})$, we need

$$
\begin{aligned}
& (4 n-3) \times\left\lceil\log _{2}(k+1)\right\rceil
\end{aligned}
$$

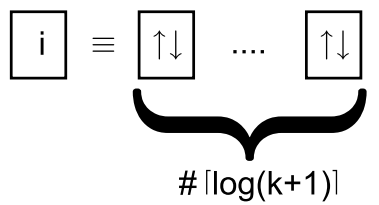

Figure 12: The $i$ th quantum number is encoded into a register made of $\lceil\log (k+1)\rceil$ qubits. 


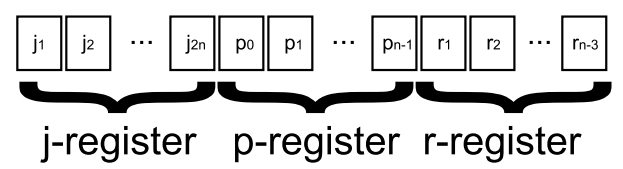

Figure 13: The register of the quantum circuit encoding an odd-basis state $|\mathbf{p} ; \mathbf{r}\rangle^{\mathbf{j}}$.

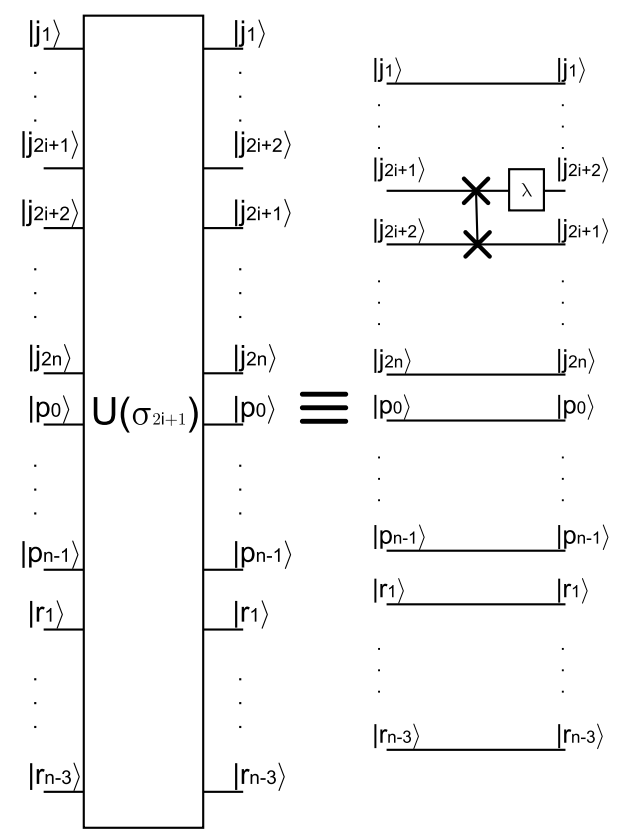

Figure 14: Circuit diagram of the unitary gate associated to an odd braid generator.

qubits to encode one basis vector. The ordering on such quantum register is shown in figure 13, where the three sets of quantum numbers are associated with a $\mathbf{j}$-register, a $\mathbf{p}$-register, and a $\mathbf{r}-$-register, respectively.

The unitary braiding operator associated with the $2 n$-strands unoriented braid under consideration can be decomposed - following the scheme explained in Section 2.2 and updating the representation according to [29] into "elementary" odd braidings and duality transformation.

The elementary braiding matrices are diagonal in the odd-coupled basis, so that their action can be easily implemented on the quantum register of figure 13. The quantum gate realization of $U\left(\sigma_{2 l+1}\right)$, shown in figure 14, is simply the identity matrix on the $(\mathbf{p}, \mathbf{r})$-registers, while the $\mathbf{j}$-register is 
modified by the action of a SWAP gate and a phase gate with a phase factor given by the eigenvalue $\lambda\left(j_{2 l+1}, j_{2 l+2}\right)$ in equation $(3.6) .^{7}$

The duality transformation introduced formally in (2.14) must be applied whenever an even elementary braiding matrix $U\left(\sigma_{2 l}\right)$ is encountered in order to recover the current, odd-coupled basis. The associated ( $q$-deformed) $3 n j$ coefficient can be split into a sequence of "elementary" duality transformations according to

$$
\begin{aligned}
A_{(\mathbf{p} ; \mathbf{r})}^{(\mathbf{q} ; \mathbf{s})}\left[\begin{array}{cc}
j_{1} & j_{2} \\
j_{3} & j_{4} \\
\vdots & \vdots \\
j_{2 n-1} & j_{2 n}
\end{array}\right]= & \sum_{t_{1} \ldots t_{n-2}} \prod_{i=1}^{n-2}\left(A_{t_{i} p_{i}}\left[\begin{array}{cc}
r_{i-1} & j_{2 i+1} \\
j_{2 i+2} & r_{i}
\end{array}\right] A_{t_{i} s_{i-1}}\left[\begin{array}{cc}
t_{i-1} & q_{i} \\
s_{i} & j_{2 n}
\end{array}\right]\right) \\
& \times \prod_{l=0}^{n-2} A_{r_{l} q_{l+1}}\left[\begin{array}{cc}
t_{l} & j_{2 l+2} \\
j_{2 l+3} & t_{l+1}
\end{array}\right]
\end{aligned}
$$

(see figure 7 of Section 2.2 for the graphical representation of such decomposition).

Each symbol in the latter expression is the matrix form of a $q-6 j$ coefficient (see figure 8), namely

$$
A_{j_{23}}^{j_{12}}\left[\begin{array}{cc}
j_{1} & j_{2} \\
j_{3} & j
\end{array}\right] \doteq(-)^{\left(j_{1}+j_{2}+j_{3}+j\right)}\left(\left[2 j_{12}+1\right]_{q}\left[2_{23}+1\right]_{q}\right)^{1 / 2}\left\{\begin{array}{ccc}
j_{1} & j_{2} & j_{12} \\
j_{3} & j & j_{23}
\end{array}\right\},
$$

where the labels of the quantum numbers has been slightly changed to comply with the following standard explicit expression of the $q-6 j$

$$
\begin{aligned}
& \left\{\begin{array}{ccc}
j_{1} & j_{2} & j_{12} \\
j_{3} & j & j_{23}
\end{array}\right\}_{q} \\
& =\Delta\left(j_{1}, j_{2}, j_{12}\right) \Delta\left(j_{3}, j, j_{12}\right) \Delta\left(j_{1}, j, j_{23}\right) \Delta\left(j_{2}, j_{3}, j_{23}\right)
\end{aligned}
$$

${ }^{7}$ Recall that a SWAP acting on two qubits $|x\rangle,|y\rangle$ is the operation

$$
\text { SWAP : }|x\rangle|y\rangle \longmapsto|y\rangle|x\rangle,
$$

which corresponds to the matrix

$$
\mathrm{SWAP}=\left(\begin{array}{cccc}
1 & 0 & 0 & 0 \\
0 & 0 & 1 & 0 \\
0 & 1 & 0 & 0 \\
0 & 0 & 0 & 1
\end{array}\right)
$$




$$
\begin{aligned}
& \times \sum_{z \geq 0}\left[\frac{(-)^{z}[z+1]_{q} !}{\left[z-j_{1}-j_{2}-j_{12}\right]_{q} !\left[z-j_{3}-j-j_{12}\right]_{q} !\left[z-j_{1}-j-j_{3}\right]_{q} !}\right. \\
& \times \frac{1}{\left[z-j_{2}-j_{3}-j_{23}\right]_{q} !\left[j_{1}+j_{2}+j_{3}+j-z\right]_{q} !} \\
& \left.\times \frac{1}{\left[j_{1}+j_{3}+j_{12}+j_{23}-z\right]_{q} !\left[j_{2}+j+j_{12}+j_{23}-z\right]_{q} !}\right] .
\end{aligned}
$$

The symbols $\Delta(\ldots)$ are combinatorial factors involving the factorials of $q$-dimensions (see Appendix I of [28]), where the factorial of a $q$-number $[x]_{q}$ is defined as $[x]_{q} !=[x]_{q}[x-1]_{q} \ldots[2]_{q}[1]_{q}$.

For each choice of the entries (in the allowed set $\{0,1 / 2, \ldots, k / 2\}$ ) the latter power series is actually a summation over the finite set of (integer and half-integers) $z$ 's that yields non-negative quantum integers.

From the remarks above it should be clear that the problem of efficiently implementing (3.13), namely the most general change of basis, is equivalent to the simpler problem of efficiently compiling a sequence of $q-6 j$ symbols or elementary duality matrices (3.14). But any such coefficient (i.e., the symbol with a fixed set of entries) can be easily and efficiently evaluated with a classical computer owing to the finiteness of its explicit expression (3.15).

On the other hand, it is necessary to make explicit the action of the matrix (3.14) on the qubit register, namely on an Hilbert space of dimension $2^{\lceil\log (k+1)\rceil}$ for each admissible set of entries. ${ }^{8}$

The circuit realization is shown in figure 15 , where the $\mathbf{j}$-register acts as a control register on the qubits of the $\mathbf{p}(\mathbf{r})$-registers involved in the transformation. The transformation $A$ can then be thought of as a gate with a "block structure", or a multiplexor associated with the block structure depicted in figure 16. Each block corresponds to a particular configuration of the $\mathbf{j}$-qubits, and the matrix element inside the block are $q-6 j$, up to suitable factors (equation (3.14)).

On the basis of the decomposition in (3.13) we realize that the allowed elementary duality matrices are always parameterized by the set $\mathbf{j}$ of those quantum numbers which remain unchanged when they are applied to the

\footnotetext{
${ }^{8}$ The "classical" $6 j$-symbol can be expressed in terms of a series similar to (3.15), but the encoding of such symbols on qubit registers would depend explicitly on the values of the entries, so that the computation is not obviously efficient with respect to the size of the input data, see footnote 2 in Section 2.1.
} 


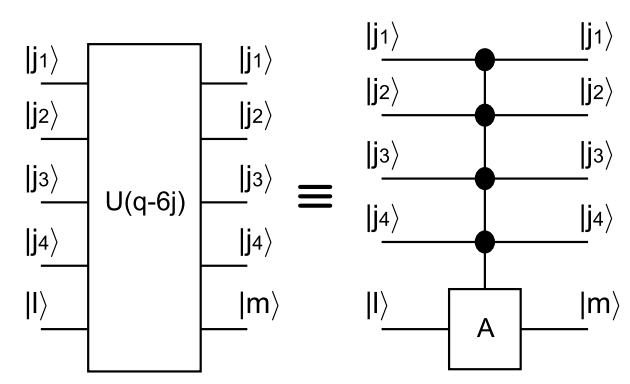

Figure 15: The multiplexor gate representing the action of a $q-6 j$ transformation.

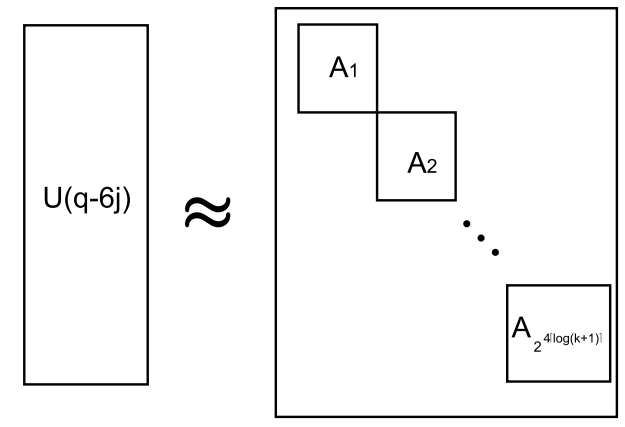

Figure 16: The block-decomposition of the matrix corresponding to a $q-6 j$ transformation.

proper registers. The crucial remark here consists in noticing that the dimension of these matrices is independent of the size of the problem, determined by the index of the braid group and the number of crossings. Since there exist efficient methods to approximate unitary matrices of a given dimension [71], a sequence of universal gates can be always worked out that efficiently approximate every $q-6 j$ as well (see [72] for more details).

The number of elementary duality transformations needed to decompose a general duality transformation (2.6) is $(2 n-3)$, linear in the size of the problem under consideration. The action of a $q-3 n j$ recoupling transformation on the register of figure 13 is shown in figure 17. Note however that, once all the $(2 n-3)$ gates represented by $q-6 j$ have been applied, it is necessary to swap some of the qubits in order to recover the proper order in the register, see figure 18 .

Upon applying a $q-3 n j$ recoupling transformation we end up in the evencoupled basis $|\mathbf{q} ; \mathbf{s}\rangle^{\mathbf{j}}$ (see (2.13) and figure 6) which diagonalizes the even braiding matrices $U\left(\sigma_{2 l}\right)$ of the Kaul representation. Their action on the (even) qubit register can then be implemented by paralleling the procedure described in the odd case. 


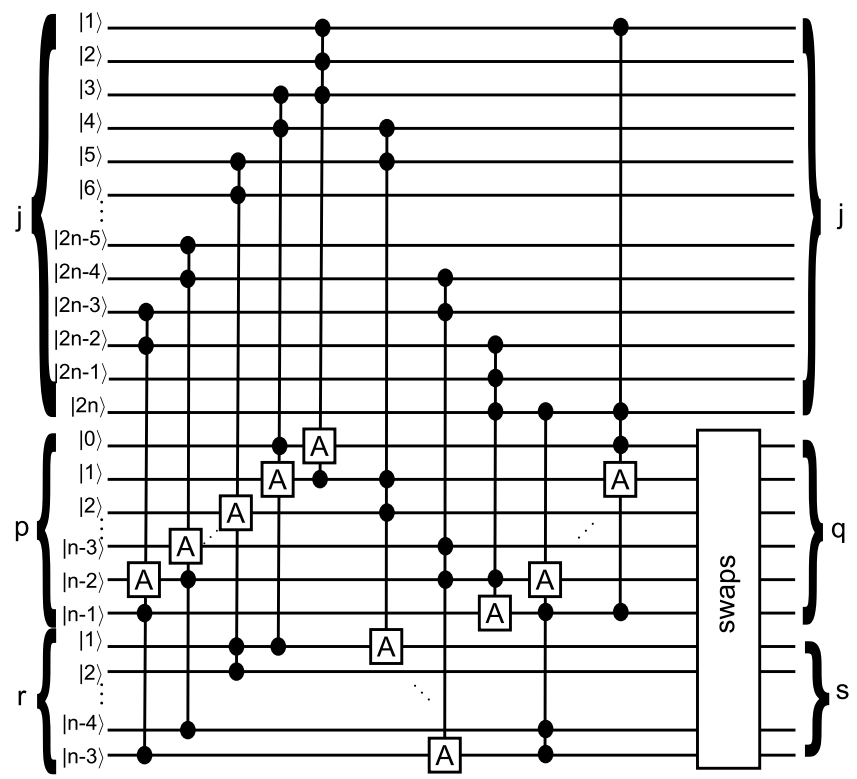

Figure 17: The circuit realization of a general $q-3 n j$ recoupling transformation.

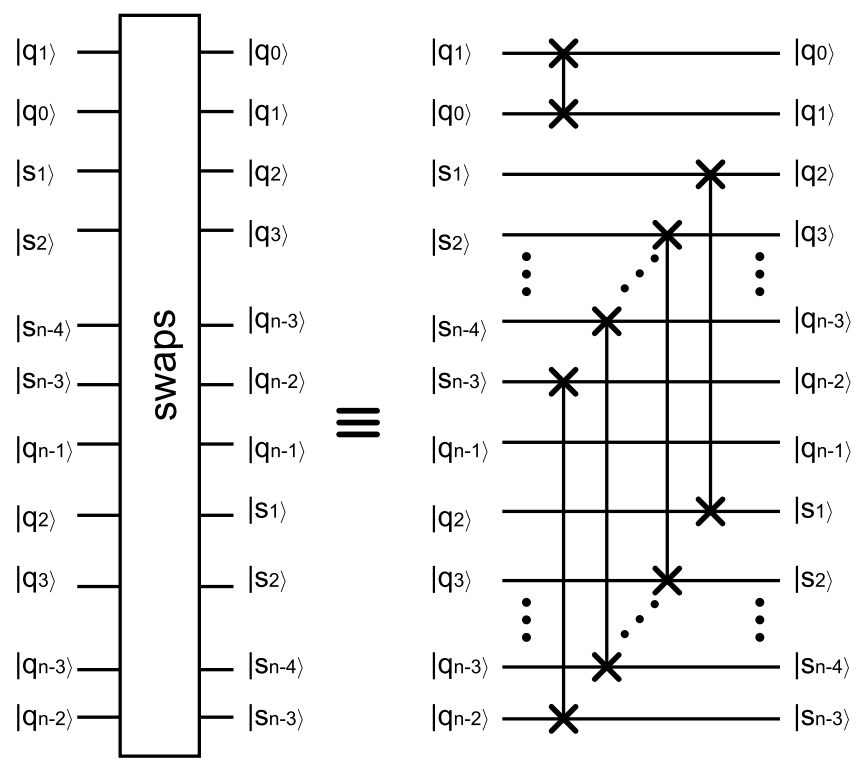

Figure 18: The sequence of SWAP gates needed to reordering the qubit register. 


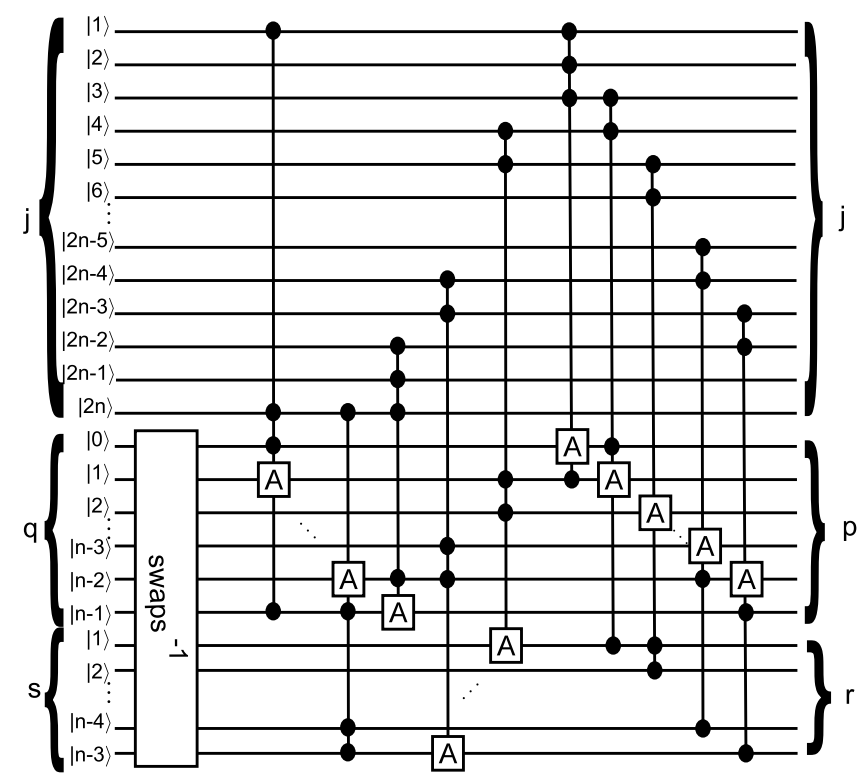

Figure 19: The inverse of the circuit of figure 17.

The further step consists in going back to the $|\mathbf{p} ; \mathbf{r}\rangle^{\mathbf{j}}$ basis, which can be achieved by means of the finite sequence of $q-6 j$ transformations shown in figure 19, eventually completed by a suitable sequence of SWAPS to recover the initial ordering of the start register.

This completes the analysis of the quantum circuit which implements efficiently the Kaul representation [29] on the Hilbert space spanned by the odd-coupled basis of conformal blocks (point ii) at the beginning of this section).

For what concerns the preparation of the start state according to point (i), notice once more that we have considered so far the case of an unoriented link $[L ; \mathbf{j}]$ (plat closure of a $2 n$-strands braid) with a fixed coloring set $\mathbf{j}$. As already pointed out in the case of oriented links (see Section 2.2, (2.15) and Appendix B) the start state of the automaton calculation is a singlet vector in the odd-coupled basis

$$
[L ; \mathbf{j}] \leftrightarrow|\mathbf{0} ; \mathbf{0}\rangle^{\mathbf{j}} ; \quad \mathbf{p}=p_{0}, \ldots, p_{n-1} \equiv \mathbf{0}, \quad \mathbf{r}=r_{0}, \ldots, r_{n-3} \equiv \mathbf{0}
$$

which can be efficiently encoded into the qubit register of figure 13 as a particular case of the construction carried out above. Then the polynomial for an unoriented, framed link $J[L ; \mathbf{f}, \mathbf{j} ; q]$ can be recovered as the (trace of the) expectation value of the associated braiding operator in the updated Kaul 
representation. However, the start state $|\psi\rangle^{\mathcal{I}}$ of the recognizer $\mathcal{A}_{q}$ needed for processing the three-manifold invariant $\mathcal{I}\left[\mathcal{M}_{L}^{3} ; \mathbf{f} ; q\right]$ must be prepared in order to comply with the summation over the colorings in (3.9). The goal can be achieved in a quite staightforward way by resorting to quantum parallelism, as described in the following.

As before, we first split the qubit register into three ordered parts, the $\mathbf{j}-, \mathbf{p}^{-}$and $\mathbf{r}-$-registers. Then we initialize the $\mathbf{j}-$-register into the following weighted superposition of states

$$
|\psi\rangle^{J} \doteq \sum_{\{\mathbf{j}\}} \sqrt{\mu_{\mathbf{j}}}|\mathbf{j}\rangle
$$

with $\mu_{\mathbf{j}} \equiv \mu_{j_{1}} \cdots \mu_{j_{S}}$ (S in the number of link components and the coefficients $\mu$ 's are defined as in theorem 3.2 of Section 3.1). Since the link $L$ can be considered as the plat closure of a braid word $w$, the quantum circuit needed to encode the start state can be implemented by the following composition of unitary gates:

$$
S^{J}(w) U^{\mathrm{J} \otimes \mathrm{P} \otimes \mathrm{R}}(w):|\psi\rangle^{J} \otimes|\mathbf{0} ; \mathbf{0}\rangle \mapsto|\psi\rangle^{J} \otimes\left|\mathbf{p}^{\prime} ; \mathbf{r}^{\prime}\right\rangle^{P \otimes R}
$$

where $S^{J}(w)$ is the SWAP gate on the $\mathbf{j}$-register and $\left|\mathbf{p}^{\prime} ; \mathbf{r}^{\prime}\right\rangle$ is the state obtained from the application of the circuit $U(w)$.

The following sequence of equalities shows that this quantum circuit actually gives the required three-manifold invariant

$$
\begin{aligned}
\mathrm{P} \otimes \mathrm{R} & \left\langle\mathbf{0} ; \mathbf{0}\left|\otimes{ }^{\mathrm{J}}\left\langle\psi\left|S^{\mathrm{J}}(w) U^{\mathrm{J} \otimes \mathrm{P} \otimes \mathrm{R}}(w)\right| \psi\right\rangle^{\mathrm{J}}\right| \mathbf{0} ; \mathbf{0}\right\rangle^{\mathrm{P} \otimes \mathrm{R}} \\
& ={ }^{\mathrm{P} \otimes \mathrm{R}}\left\langle\mathbf{0} ; \mathbf{0}\left|\otimes{ }^{\mathrm{J}}\langle\psi|\cdot| \psi\rangle^{\mathrm{J}} \otimes U^{\mathrm{P} \otimes \mathrm{R}}(w)\right| \mathbf{0} ; \mathbf{0}\right\rangle^{\mathrm{P} \otimes \mathrm{R}} \\
& =\sum_{\{\mathbf{j}\}} \mu_{\mathbf{j}}\left\langle\mathbf{0} ; \mathbf{0}\left|U^{\mathrm{P} \otimes \mathrm{R}}(w)\right| \mathbf{0} ; \mathbf{0}\right\rangle \\
& =\mathcal{I}\left[\mathcal{M}_{L}^{3} ; \mathbf{f} ; q\right] \alpha^{\sigma[L ; \mathbf{f}]} .
\end{aligned}
$$

Recall that the signature of the linking matrix $\sigma[L ; \mathbf{f}]$ can be easily computed classically once the linking matrix (3.8) (which is part of the topological input data of the algorithmic problem) is given.

Finally, the efficient sampling of the value of the invariant referred to in point iii) can be carried out by resorting to the techniques of $[15,16]$, as already done in [3] for the case of colored link polynomials. 


\section{Concluding remarks}

We have shown that all the significant quantities - partition functions and observables - in SU(2) quantum CSW theory can be efficiently approximated by quantum algorithms at finite values of the coupling constant $k$, reflecting the intrinsic field-theoretic solvability of this theory. The efficiency of the quantum algorithms is proved on the basis of a two-level computational scheme which relies on the implementation of unitary representations of the braid group proposed in $[28,29]$. In particular, as shown in Section 3.2, the representation needed to handle three-manifold quantum invariants can be efficiently compiled on a quantum circuit equipped with a suitable start qubit register, namely within the "standard" model of quantum computation.

The relevance of our result in connection with the algorithmic complexity of quantum CSW theory has been extensively addressed in Section 1.2, while further developments and applications to geometry and quantum gravity models had already been discussed in Section 1.3.

More generally, it would be interesting to improve our approach, on the one hand, by handling quantum topological invariants arising from CSW theories with arbitrary semi-simple Lie groups [73] and, on the other, by exploring the quantum computational complexity of solvable models in statistical mechanics [74].

Finally, let us comment in some more details the issue concerning the model of quantum computation adopted. As pointed out in $(\mathbf{A})$ and $(\mathbf{C})$ of Section 3.2, a central role in our procedure is played by the quantum recognizer $\mathcal{A}_{q}$ able to process efficiently the language generate by the braid group with transition matrices given by Kaul unitary representations (Sections 2.2 and 3.2) and probability distributions associated with quantum topological invariants. It may be tempting to proceed without this step, processing directly the unitary representations within the quantum circuit scheme of computation. However, we proved in Section 3.2 that the basic morphisms of the $q$-tensor category $\left(\mathfrak{R}\left(S U(2)_{q}\right) ; \mathcal{R} ; \mathcal{F}\right)$ on which the recognizer is modelled can be efficiently compiled and approximated on a quantum circuit (in particular the implementation of a $q-6 j$ transformation is independent of both the input size of the algorithmic problem and on the values of its entries; see also footnote 2, Sections 2.1, 2.7 and Section 3.2).

This achievement, quite remarkable as it is by itself, opens as well the further possibility of looking at the $q$-spin network simulator as the fundamental model of computation for a wide range of algorithmic problems in geometric topology and group theory. According to the quantum recognizer 
definition given in Section 2.1, the specific problems that can be dealt with it require the selection of suitable start states - that must be efficiently encoded into qubit registers - and of (possibly constrained) sets of transition matrices. We conjecture that the approximate evaluation of invariants for (colored) triangulations in dimensions 2 and 3 should require minor modifications of the scheme employed in this paper for processing quantum invariants of links and three-manifolds.

\section{Acknowledgments}

We are in debt with Romesh Kaul for clarifying remarks on his work and for suggesting us possible further developments.

\section{Appendix A The spin network quantum simulator}

The universal model of quantum computation proposed in [41-43] relies on the (re)coupling theory of $\mathrm{SU}(2)$ angular momenta [56,60]. It can be thought of as a generalization of the standard quantum circuit model in which the computational Hilbert spaces are binary-coupled eigestates of $N \equiv(n+1) \mathrm{SU}(2)$-angular momenta (whose quantum numbers range over $\{0,1 / 2,1,3 / 2, \ldots\})$ and unitary transformations ("gates") are expressed in terms of recoupling coefficients ( $3 n j$ symbols) connecting pairs of inequivalent binary coupling schemes. ${ }^{9}$

The architecture of the spin network is modelled as an $\mathrm{SU}(2)$ fiber space structure over a discrete base space $V$

$$
\left(V, \mathbb{C}^{2 J+1}\right)_{n}
$$

which encodes all possible computational Hilbert spaces as well as all gates for any fixed number $N=n+1$ of incoming angular momenta (see Appen$\operatorname{dix} \mathrm{A}$ of [42] and [43] for more details). The base space $V \doteq\{v(\mathfrak{b})\}$ represents the vertex set of a regular, three-valent graph, the so-called twist-rotation graph [67] $\mathfrak{G}_{n}(V, E)$ with cardinality $|V|=(2 n) ! / n$ !, i.e., the quadruple factorial number. $E$, the edge set of the graph, will be associated with permitted transformations between pairs of verices as described below.

There exists a 1:1 correspondence

$$
\{v(\mathfrak{b})\} \longleftrightarrow\left\{\mathcal{H}_{n}^{J}(\mathfrak{b})\right\}
$$

\footnotetext{
${ }^{9}$ The model can be extended to include Wigner rotations in the eigenspace of the total angular momentum, see Section 3.2 of [42].
} 
between the vertices of $\mathfrak{G}_{n}(V, E)$ and the computational Hilbert spaces of the simulator, where $\mathfrak{b}$ denotes the binary bracketing structure that we are going to describe. For a given value of $n, \mathcal{H}_{n}^{J}(\mathfrak{b})$ is the simultaneous eigenspace of the squares of $2 n+1$ Hermitean, mutually commuting angular momentum operators

$$
\begin{aligned}
& \mathbf{J}_{1}, \mathbf{J}_{2}, \mathbf{J}_{3}, \ldots, \mathbf{J}_{n+1} \equiv\left\{\mathbf{J}_{i}\right\} ; \quad \mathbf{J}_{1}+\mathbf{J}_{2}+\mathbf{J}_{3}+\cdots+\mathbf{J}_{n+1} \doteq \mathbf{J} \\
& \text { and } \quad \mathbf{K}_{1}, \mathbf{K}_{2}, \mathbf{K}_{3}, \ldots, \mathbf{K}_{n-1} \equiv\left\{\mathbf{K}_{h}\right\}
\end{aligned}
$$

together with the operator $J_{z}$ (the projection of the total angular momentum $\mathbf{J}$ along the quantization axis). The associated quantum numbers are $j_{1}, j_{2}, \ldots, j_{n+1} ; J ; k_{1}, k_{2}, \ldots, k_{n-1}$ and $M$, where $-J \leq M \leq$ in integer steps. If $\mathcal{H}^{j_{1}} \otimes \mathcal{H}^{j_{2}} \otimes \cdots \otimes \mathcal{H}^{j_{n}} \otimes \mathcal{H}^{j_{n+1}}$ denotes the factorized Hilbert space, namely the $(n+1)$-fold tensor product of the individual eigenspaces of the $\left(\mathbf{J}_{i}\right)^{2}$ 's, the operators $\mathbf{K}_{h}$ 's represent intermediate angular momenta generated, through Clebsch-Gordan series, whenever a pair of $\mathbf{J}_{i}$ 's is coupled.

As an example, by coupling sequentially the $\mathbf{J}_{i}$ 's according to the scheme $\left(\cdots\left(\left(\mathbf{J}_{1}+\mathbf{J}_{2}\right)+\mathbf{J}_{3}\right)+\cdots+\mathbf{J}_{n+1}\right)=\mathbf{J}$ - which generates $\left(\mathbf{J}_{1}+\mathbf{J}_{2}\right)=\mathbf{K}_{1}$, $\left(\mathbf{K}_{1}+\mathbf{J}_{3}\right)=\mathbf{K}_{2}$, and so on - we would get a binary bracketing structure of the type $\left(\cdots\left(\left(\left(\mathcal{H}^{j_{1}} \otimes \mathcal{H}^{j_{2}}\right)_{k_{1}} \otimes \mathcal{H}^{j_{3}}\right)_{k_{2}} \otimes \cdots \otimes \mathcal{H}^{j_{n+1}}\right)_{k_{n-1}}\right)_{J}$, where we add an overall bracket labeled by the quantum number of the total angular momentum $J$. Note that, as far as $j_{i}$ 's quantum numbers are involved, any value belonging to $\{0,1 / 2,1,3 / 2, \ldots\}$ is allowed, while the ranges of the $k_{h}$ 's are suitably constrained by Clebsch-Gordan decompositions (e.g., if $\left(\mathbf{J}_{1}+\right.$ $\left.\left.\mathbf{J}_{2}\right)=\mathbf{K}_{1} \Rightarrow\left|j_{1}-j_{2}\right| \leq k_{1} \leq j_{1}+j_{2}\right)$. We denote a binary coupled basis of $(n+1)$ angular momenta in the $J M$-representation and the corresponding Hilbert space appearing in (A.2) as

$$
\begin{aligned}
& \left\{\left|\left[j_{1}, j_{2}, j_{3}, \ldots, j_{n+1}\right]^{\mathfrak{b}} ; k_{1}^{\mathfrak{b}}, k_{2}^{\mathfrak{b}}, \ldots, k_{n-1}^{\mathfrak{b}} ; J M\right\rangle,-J \leq M \leq J\right\} \\
& \quad=\mathcal{H}_{n}^{J}(\mathfrak{b}) \doteq \operatorname{span}\left\{|\mathfrak{b} ; J M\rangle_{n}\right\}
\end{aligned}
$$

where the string inside $\left[j_{1}, j_{2}, j_{3}, \ldots, j_{n+1}\right]^{\mathfrak{b}}$ is not necessarily ordered, $\mathfrak{b}$ is the shorthand notation for the current binary bracketing structure and the $k_{h}$ 's are uniquely associated with the chain of pairwise couplings selected by $\mathfrak{b}$.

For a given value of $J$ each $\mathcal{H}_{n}^{J}(\mathfrak{b})$ has dimension $(2 J+1)$ over $\mathbb{C}$ and thus there exists one isomorphism

$$
\mathcal{H}_{n}^{J}(\mathfrak{b}) \cong_{\mathfrak{b}} \mathbb{C}^{2 J+1}
$$


for each admissible binary coupling scheme $\mathfrak{b}$ of $(n+1)$ incoming spins. It is worth stressing that such isomorphic spaces are physically inequivalent as far as they are associated with different schemes of (binary) interactions.

The vector space $\mathbb{C}^{2 J+1}$ is interpreted as the typical fiber attached to each vertex $v(\mathfrak{b}) \in V$ of the fiber space structure (A.1) through the isomorphism (A.5).

For what concerns unitary operations acting on the computational Hilbert spaces (A.4), it can be shown [56] that any $3 n j$ symbols of $\mathrm{SU}(2)$ can be splitted into "elementary gates" represented by Racah and phase transformations (in the categorical language of Section 2.1 they are referred to as the basic morphisms introduced in (2.2) and (2.1), respectively). A Racah transform applied to a basis vector of the type (A.4) is defined formally as

$$
F:\left|\ldots\left((a b)_{d} c\right)_{f} \ldots ; J M\right\rangle \mapsto\left|\ldots\left(a(b c)_{e}\right)_{f} \ldots ; J M\right\rangle,
$$

where we are using here Latin letters $a, b, c, \ldots$ to denote both incoming $\left(j_{i} \mathrm{~s}\right.$ in the previous notation) and intermediate $\left(k_{h}\right.$ 's) spin quantum numbers. The explicit expression of (A.6) reads

$$
\begin{aligned}
& \left|\left(a(b c)_{e}\right)_{f} ; M\right\rangle \\
& \quad=\sum_{d}(-1)^{a+b+c+f}[(2 d+1)(2 e+1)]^{1 / 2}\left\{\begin{array}{ccc}
a & b & d \\
c & f & e
\end{array}\right\}\left|\left((a b)_{d} c\right)_{f} ; M\right\rangle,
\end{aligned}
$$

where there appears the Racah-Wigner $6 j$ symbol of $\mathrm{SU}(2)$ and $f$ here plays the role of the total angular momentum quantum number. Owing to the Wigner-Eckart theorem, the magnetic quantum number is not affected by such transformation and the same holds true for a general, $3 \mathrm{nj}$ recoupling coefficient. Recall also that the square of the $6 j$ symbol in (A.7) represents the probability that a system prepared in the state $\left|\left((a b)_{d} c\right)_{f} ; M\right\rangle$ will be measured in the state $\left|\left(a(b c)_{e}\right)_{f} ; M\right\rangle$.

A phase transform on a basis vector (A.4) is defined as

$$
R:\left|\ldots(a b)_{c} \ldots ; J M\right\rangle \mapsto\left|\ldots(b a)_{c} \ldots ; J M\right\rangle,
$$

and explicitly reads

$$
\left|\ldots(a b)_{c} \ldots ; J M\right\rangle=(-)^{a+b-c}\left|\ldots(b a)_{c} \ldots ; J M\right\rangle,
$$


The edge set $E=\{e\}$ of the twist-rotation graph $\mathfrak{G}_{n}(V, E)$ is a subset of the Cartesian product $(V \times V)$ where an (undirected) arc between two vertices $v(\mathfrak{b})$ and $v\left(\mathfrak{b}^{\prime}\right)$

$$
e\left(\mathfrak{b}, \mathfrak{b}^{\prime}\right) \doteq\left(v(\mathfrak{b}), v\left(\mathfrak{b}^{\prime}\right)\right) \in(V \times V)
$$

exists iff the underlying Hilbert spaces are related to each other by an elementary unitary operation of the type (A.6) or (A.8). Note also that

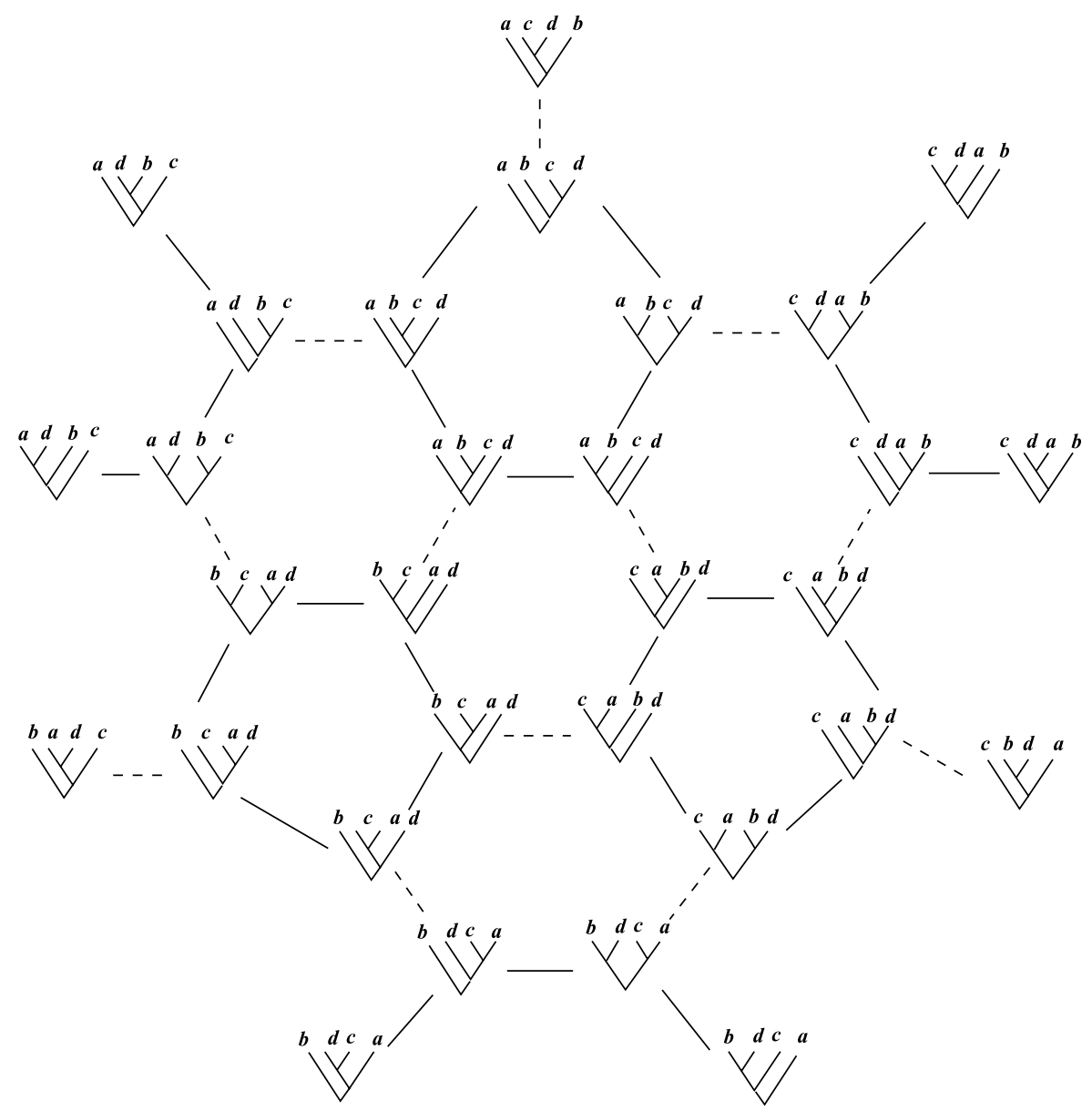

Figure 20: A portion of the spin network graph $\mathfrak{G}_{3}(V, E)$. The vertices are labelled by rooted binary trees encoding the combinatorics of the binary coupled computational Hilbert spaces while the edges represent Racah transformations and the dashed ones are phases. 
elements in $E$ can be considered as mappings

$$
\begin{gathered}
\left(V \times \mathbb{C}^{2 J+1}\right)_{n} \longrightarrow\left(V \times \mathbb{C}^{2 J+1}\right)_{n}, \\
\left(v(\mathfrak{b}), \mathcal{H}_{n}^{J}(\mathfrak{b})\right) \mapsto\left(v\left(\mathfrak{b}^{\prime}\right), \mathcal{H}_{n}^{J}\left(\mathfrak{b}^{\prime}\right)\right),
\end{gathered}
$$

connecting each given decorated vertex to one of its nearest vertices and thus define a "transport prescription in the horizontal sections" belonging to the total space $\left(V \times \mathbb{C}^{2 J+1}\right)_{n}$ of the fiber bundle (A.1).

The structure of the graph $\mathfrak{G}_{n}(V, E)$ in the case of $(n+1)=4$ incoming spin variables $a, b, c, d$ is shown in figure 20 (such a combinatorial pattern encoding both binary-coupled Hilbert spaces and transformations among them was used for the first time in [75]).

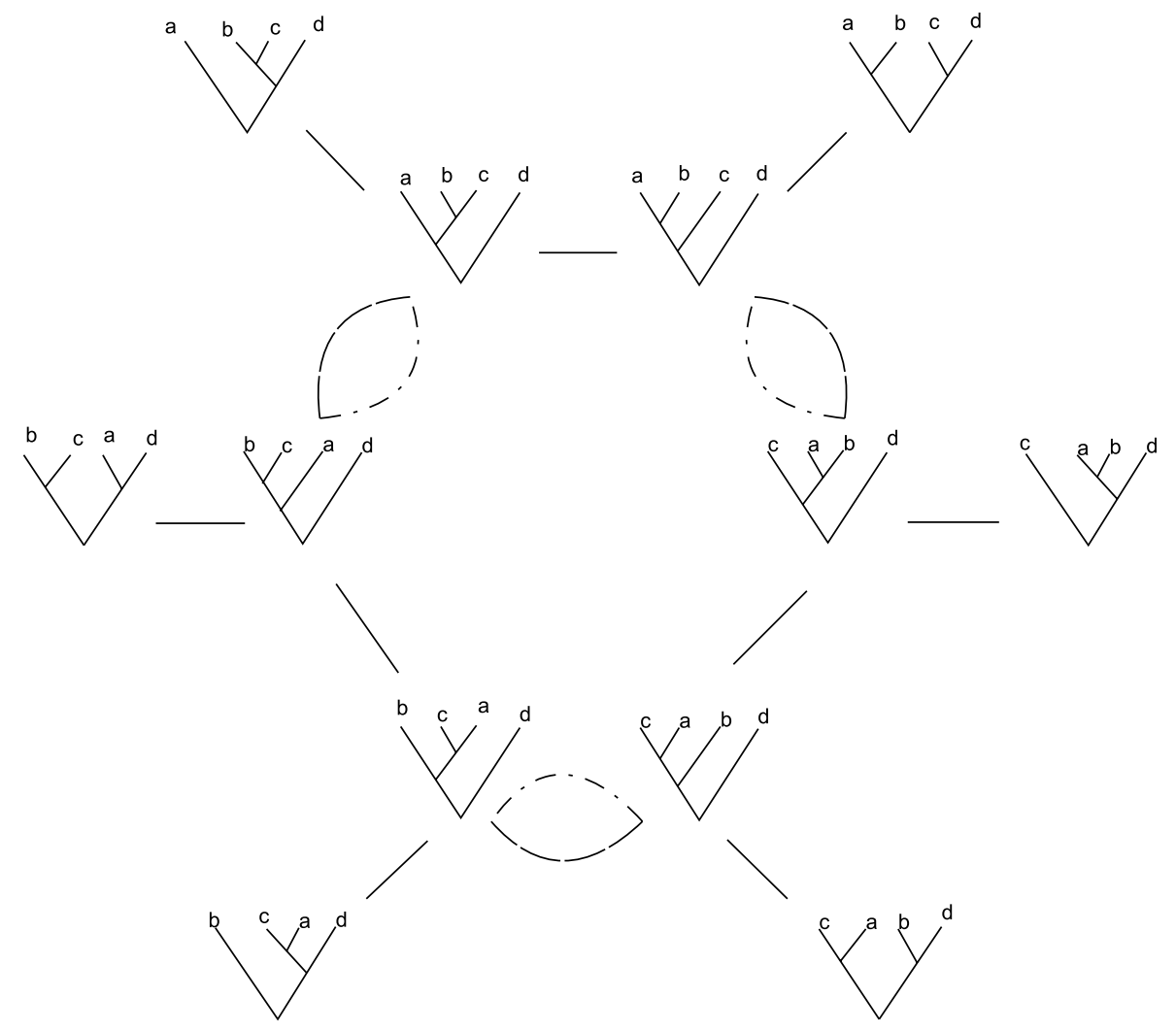

Figure 21: A portion of the $q$-deformed twist-rotation graph $\left(\mathfrak{G}_{3}(V, E)\right)_{q}$ : with respect to the previous figure, each phase transformation has been splitted in order to make manifest the non-trivial braiding features. 
The crucial feature that characterizes the graph $\mathfrak{G}_{n}(V, E)$ arises from the compatibility conditions relating the basic morphisms (A.6) and (A.8) (referred to as the hexagon and pentagon relations, see Section 2.1). The Racah identity and the Biedenharn-Elliott identity together with the orthogonality conditions for $6 j$ symbols (see, e.g., [60] for their explicit expressions) ensure that any simple path in $\mathfrak{G}_{n}(V, E)$ with fixed endpoints can be freely deformed into any other, providing identical quantum transition amplitudes at the kinematical level.

For what concerns the $q$-deformed spin network modelled on the $q$-tensor category $\left(\mathfrak{R}\left(\mathrm{SU}(2)_{q}\right) ; \mathcal{R} ; \mathcal{F}\right)$ defined in $(2.4)$ of Section 2.1 , we omit here all technical details and refer the reader to Section 4 of [3] (see also the appendix of [28] for both definitions and notations). As already pointed out, the basic morphism $\mathcal{F}$ is implemented in this case by means of a $q-6 j$ symbol defined in (3.15) while the braiding $\mathcal{R}$ is to be associated with the over- or undercrossings of two contiguous strands belonging to (colored) braids (see e.g., the eigenvalue equation (3.6) of the odd braiding operators employed in Section 3.2).

A pictorial representation of $q$-deformed spin network graph is shown in figure 21.

\section{Appendix B Automaton calculation}

The Jones polynomial [11] is a particular instance of colored link polynomial where the labels of the conformal block basis correspond to the fundamental, $\frac{1}{2}$-irrep of $\mathrm{SU}(2)_{q}$. This allows us to simplify the notation for the states of the recognizer $\mathcal{A}_{q}$ defined in Section 2.2 by setting

$$
|\mathbf{p} ; \mathbf{r}\rangle^{\mathbf{j}} \rightarrow|\mathbf{p} ; \mathbf{r}\rangle,
$$

since the coloring assignment $\{\mathbf{j}\}$ is always a string of $2 n \frac{1}{2}$-spins. The start state of the automaton is chosen to be $|\mathbf{0} ; \mathbf{0}\rangle$, i.e., all the internal labels are equal to 0 , as depicted in figure 22 .

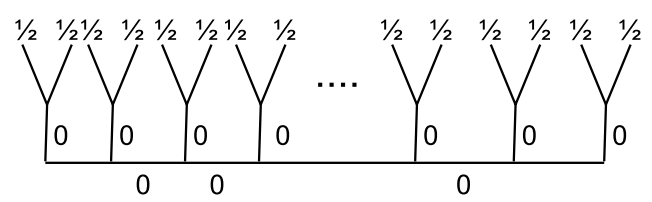

Figure 22: The start state of the $q$-spin network automaton with probability distribution associated with the Jones polynomial. 
As discussed at the end of Section 2.2, let us provide the automaton with an input word $w$ of length $\kappa$ corresponding to an unitary evolution $U(w)$ expressed in terms of the sequence given in (2.18).

In order to complete the definition of $\mathcal{A}_{q}$ we need explicit expressions for $P$ (accept) and $P$ (reject) (see Section 2.1). We choose the following:

$$
\begin{aligned}
P(\text { accept }) & \equiv|\mathbf{0} ; \mathbf{0}\rangle\langle\mathbf{0} ; \mathbf{0}|, \\
P(\text { reject }) & \equiv \mathbb{I}-|\mathbf{0} ; \mathbf{0}\rangle\langle\mathbf{0} ; \mathbf{0}| .
\end{aligned}
$$

The "a priori" probability distribution for the language generated by the braid group that we choose is the square modulus of the Jones polynomial of the plat closure $\hat{w}$ of the braid $w$, namely

$$
\operatorname{Pr}(w)=|J(\hat{w} ; q)|^{2} \equiv V(\hat{w} ; q),
$$

where $q$ is the root of unity at which the polynomial is evaluated. Using the properties of the Kaul representation (see (2.15)) and (B.1) there follows that

$$
\begin{aligned}
& \mid \operatorname{Pr}(w)-\langle\mathbf{0} ; \mathbf{0}| U^{\dagger}(w) P(\text { accept }) U(w)|\mathbf{0} ; \mathbf{0}\rangle \mid \\
& \quad=\left|\operatorname{Pr}(w)-\left\langle\mathbf{0} ; \mathbf{0}\left|U^{\dagger}(w)\right| \mathbf{0} ; \mathbf{0}\right\rangle\langle\mathbf{0} ; \mathbf{0}|U(w)| \mathbf{0} ; \mathbf{0}\rangle\right| \\
& \quad=\left.|\operatorname{Pr}(w)-|\langle\mathbf{0} ; \mathbf{0}|U(w)| \mathbf{0} ; \mathbf{0}\rangle\right|^{2} \mid \\
& \quad=\left.|V(\hat{w}, q)-|\langle\mathbf{0} ; \mathbf{0}|U(w)| \mathbf{0} ; \mathbf{0}\rangle\right|^{2} \mid=0 .
\end{aligned}
$$

Thus we have shown that the spin network quantum automaton recognizes "exactly" (namely with a word-probability threshold $\delta=0$, see $(2.8)$ in Section 2.1) the braid group language according to the "Jones probability distribution".

A similar result holds true for the families of automata $\mathcal{A}_{q}$ parametrized by $\left(j_{1}, j_{2}, \ldots, j_{2 n}\right)$ that recognize the braid group language $B_{2 n}$ with a probability distribution given by the colored polynomial (2.15).

\section{References}

[1] K. Wiesner and J. P. Crutchfield, Computation in finitary quantum processes, 2006, eprint quant-ph/0608206v4.

[2] S. Garnerone, A. Marzuoli and M. Rasetti, Spin networks, quantum automata and link invariants, J. Phys.: Conf. Ser. 33 (2006), 95; Quantum computation of universal link invariants, Open Sys. Inf. Dyn. 
13 (2006), 373; Quantum automata, braid group and link polynomials, Quant. Inf. \& Comp. 7(526) (2007), 479, eprint quant-ph/0601169.

[3] S. Garnerone, A. Marzuoli and M. Rasetti, An efficient quantum algorithm for colored Jones polynomials, Int. J. Quant. Inf. 6(Suppl.) (2008), 773, eprint quant-ph/0606167;

Quantum geometry and quantum algorithms, J. Phys. A: Math. Theor. 40 (2007), 3047, eprint quant-ph/0607203.

[4] M. H. Freedman, $P / N P$ and the quantum field computer, Proc. Natl. Acad. Sci. USA 95 (1998), 98.

[5] H. M. Freedman, A. Kitaev and Z. Wang, Simulation of topological field theories by quantum computer, Commun. Math. Phys. 227 (2002), 587.

[6] H. M. Freedman, M. Larsen and Z. Wang, A modular functor which is universal for quantum computation, Commun. Math. Phys. 227 (2002), 605.

[7] J. Preskill, Topological quantum computing for beginners, 2004 (http://online.kitp.ucsb.edu.online/exotic_c04/preskill/).

[8] E. Witten, Quantum field theory and the Jones polynomial, Commun. Math. Phys. 121 (1989), 351.

[9] M. F. Atiyah, Topological quantum field theories, Publ. Math. IHES 68 (1989), 175.

[10] D. Birmingham, M. Blau, M. Rakowski and G. Thomson, Topological field theory, Phys. Rep. 209 (1991), 129.

[11] V. F. R Jones, A polynomial invariant of knots via von Neummann algebras, Bull. Amer. Math. Soc. 12 (1985), 103;

Hecke algebra representations of braid groups and link polynomials, Ann. Math. 126 (1987), 335.

[12] N. Reshetikhin and V. G. Turaev, Invariants of 3-manifolds via linkpolynomials and quantum groups, Invent. Math. 103 (1991), 547.

[13] M. A. Nielsen and I. L. Chuang, Quantum computation and quantum information, Cambridge University Press, 2000.

[14] M. Bordewich, M. Freedman, L. Lovász and D. Welsh, Approximate counting and quantum computation, Comb. Probab. Comput. 14 (2005), 737.

[15] D. Aharonov, V. Jones and Z. Landau, A polynomial quantum algorithm for approximating the Jones polynomial, 2005, eprint quant-ph/0511096v2.

[16] P. Wocjan and J. Yard, The Jones polynomial: quantum algorithms and applications in quantum complexity theory, 2006, eprint quant-ph/0603069v3. 
[17] V. G. Turaev, Quantum invariants of knots and 3-manifolds, de Gruyter, Berlin, 1994.

[18] Drinfel'd, Quantum groups, in Proc. of ICM 1986, Amer. Math. Soc. Providence, RI, 1987, 798.

[19] M. Jimbo, A q-difference analogue of $U(g)$ and the Yang Baxter equation, Lett. Math. Phys. 10 (1985), 63;

A q-analogue of $U(g)(N+1)$, Hecke algebra and the Yang Baxter equation, Lett. Math. Phys. 11 (1986), 247.

[20] F. Y. Wu, Knot theory and statistical mechanics, Rev. Mod. Phys. 64 (1992), 1099.

[21] T. Ohtsuki, ed. Problems on invariants of knots and 3-manifolds, RIMS Geometry and Topology Monographs, 4, 2002, eprint math.GT/0406190.

[22] R. K. Kaul, T. R. Govindarajan and P. Ramadevi, Schwarz type topological quantum field theories, Encycl. Math. Phys. Elsevier, Amsterdam, 2005, eprint hep-th/0504100.

[23] E. Guadagnini, The link invariants of the Chern-Simons field theory, de Gruyter, Berlin, 1993.

[24] T. Kohno, Hecke algebra representations of braid groups and classical Yang-Baxter equations, Adv. Studies Pure Math. 16 (1988), 255.

[25] C. Gomez, M. Ruiz-Altaba and G. Sierra, Quantum group in twodimensional physics, Cambridge University Press, 1996.

[26] S. Carlip, Quantum gravity in $2+1$ dimensions, Cambridge University Press, 1998.

[27] P. Ramadevi, T. R. Govindarajan and R. K. Kaul, Representations of composite braids and invariants for mutant knots and links in ChernSimons field theories, Mod. Phys. Lett. A9 (1994), 3205.

[28] R. K. Kaul, Chern-Simons theory, colored-oriented braids and link invariants, Commun. Math. Phys. 162 (1994), 289.

[29] R. K. Kaul, Chern-Simons theory, knot invariants, vertex models and three-manifold invariants, in "Horizons in World Physics", 227, Nova Science Publ. New York, 1999, eprint hep-th/9804122.

[30] R. Kirby and P. Melvin, The 3-manifold invariant of Witten and Reshetikhin-Turaev for sl(2,C), Invent. Math. 105 (1991), 473.

[31] M. R. Garey and D. S. Johnson, Computers and intractability, a guide to the theory of NP-completeness, Freeman and Co., New York, 1979.

[32] M. Freedman, C. Nayak and K. Shtengel, An extended Hubbard model with ring exchange: a route to a non-Abelian topological phase, Phys. Rev. Lett. 94 (2005), 066401. 
[33] C. Zhang, V. W. Scarola, S. Tewari and S. Das Sarma, Anyonic braidings in optical lattices, 2006, eprint quant-ph/0609101.

[34] J. S. Birman and T. E. Brendle, Braids: a survey, in "Handbook of Knot Theory", eds. W. Menasco and Thistlethwaite, Elsevier, Amsterdam, 2005. eprint math. GT/0409205.

[35] F. Jaeger, D. L. Vertigan and D. J. A. Welsh, On the computational complexity of the Jones and Tutte polynomials, Math. Proc. Camb. Phil. Soc. 108 (1990), 35.

[36] V. Subramanian and P. Ramadevi, Quantum computation of Jones polynomial, 2002, eprint quant-ph/0210095v2.

[37] L. H. Kauffman and S. J. Lomonaco, Topological quantum computing and the Jones polynomial, Proc. SPIE 6244 (2006), 62440Z. eprint quant-ph/0605004;

q-deformed spin networks, knot polynomials and anyonic topological quantum computation, 2006, eprint quant-ph/0606114v2.

[38] R. P. Feynman, Simulating physics with computers, Int. J. Theor. Phys., 21 (1982), 467.

[39] S. Lloyd, A theory of quantum gravity based on quantum computation, 2005, eprint quant-ph/0501135v8.

[40] P. A. Zizzi, Quantum computing spacetime, 2002, eprint gr-qc/0204007; Spacetime at the Planck scale: the quantum computer view, 2003, eprint gr-qc/0304032v2;

Computability at the Planck scale, 2004, eprint gr-qc/0412076v2.

[41] A. Marzuoli and M. Rasetti, Spin network quantum simulator, Phys. Lett. A306 (2002), 79.

[42] A. Marzuoli and M. Rasetti, Computing spin networks, Ann. Phys. 318 (2005), 345 .

[43] A. Marzuoli and M. Rasetti, Spin network setting of topological quantum computation, Int. J. Quant. Inf. 3 (2005), 65.

[44] A. Marzuoli and M. Rasetti, Coupling of quantum angular momenta: an insight into analogic/discrete and local/global models of computation, Nat. Comput. 6 (2007), 151, DOI: 10.1007/s11047-006-9018-4, 2006, eprint cs.CC/0610171.

[45] G. Ponzano and T. Regge, Semiclassical limit of Racah coefficients, in 'Spectroscopic and Group Theoretical Methods in Physics', eds. F. Bloch et al., North-Holland, Amsterdam, 1968, 1.

[46] R. Penrose, Angular momentum: an approach to combinatorial spacetime, in 'Quantum Theory and Beyond', ed. T. Bastin, Cambridge University Press, 1971, 151. 
[47] V. G. Turaev and Ya Viro O, State sum invariants of 3-manifolds and quantum 6j-symbols, Topology 31 (1992), 865.

[48] R. Gambini and J. Pullin, Loops, knots, gauge theories and quantum gravity, Cambridge University Press, 1996.

[49] C. Rovelli, Quantum gravity, Cambridge University Press, 2004.

[50] R. Gambini, J. Griego and J. Pullin, Chern-Simons states in spinnetwork quantum gravity, Phys. Lett. B413 (1997), 260;

A spin network generalization of the Jones Polynomial and Vassiliev invariants, Phys. Lett. B425 (1998), 41.

[51] R. M. Kashaev, A link invariant from quantum dilogarithm, Mod. Phys. Lett. A10 (1995), 1409.

[52] H. Murakami and J. Murakami, The colored Jones polynomial and the simplicial volume of a knot, Acta Math. 186 (2001), 85.

[53] D. Aharonov and I. Arad, The BPQ-hardness of approximating the Jones polynomial, 2006, eprint quant-ph/0605181v2.

[54] S. Carlip, A note on real tunnelling geometries, Class. Quant. Grav. 22 (2005), 4381.

[55] J. B. Hartle and S. W. Hawking, Wave function of the Universe, Phys. Rev. D28 (1983), 2960.

[56] L. C. Biedenharn and J. D. Louck, The Racah-Wigner algebra in quantum theory, in "Encyclopedia of Mathematics and its Applications", 9, ed. G-C. Rota, Addison-Wesley, Reading, MA, 1981.

[57] J. Fuchs and C. Schweigert, Symmetries, Lie algebras and representation, Cambridge University Press, 1997.

[58] W. P. Joyce, Diagram projection rules for recoupling diagrams in the Racah-Wigner category, J. Math. Phys. 42 (2001), 1346;

Recoupling Lie algebra and universal w-algebra, J. Math. Phys. 45 (2004), 3859.

[59] A. Joyal and R. Street, Braided tensor categories, Adv. Math. 102 (1993), 20.

[60] D. A. Varshalovich, A. N. Moskalev and V. K. Khersonskii, Quantum theory of angular momentum, World Scientific, Singapore, 1988.

[61] S. Garnerone, A. Marzuoli and M. Rasetti, Quantum knitting, Laser Phys. 16 (2006), 1582.

[62] M. H. Freedman and Z. Wang, Large Fourier transform never exactly realized by braiding conformal blocks, 2006, eprint cond-mat/0609411v2. 
[63] D. Bacon, A. Harrow and I. L. Chuang, Efficient quantum circuits for Schur and Clebsch-Gordan transforms, Phys. Rev. Lett. 97 (2006), 170502.

[64] J. S. Birman, Braids, links and mapping class groups, Princeton Univ. Press, Princeton, NJ, 1974.

[65] P. Zanardi and M. Rasetti, Holonomic quantum computation, Phys. Lett. A264 (1999), 94.

[66] J. Pachos, P. Zanardi and M. Rasetti, Non-Abelian Berry connections for quantum computation, Phys. Rev. A61 (2000), 010305(R).

[67] V. Fack, S. Lievens and J. Van der Jeugt, On the diameter of the rotation graph of binary coupling trees, Discr. Math. 245 (2002), 1.

[68] W. B. R. Lickorish, An introduction to knot theory, Springer, New York, 1997.

[69] W. B. R. Lickorish, A representation of orientable combinatorial 3manifolds, Ann. Math. 76 (1962), 531.

[70] A. Wallace, Modifications and cobounding manifolds, Canad. J. Math. 12 (1960), 503.

[71] V. V. Shende, S. S. Bullock and I. L. Markov, Trans. Computer-Aided Design 25 (2006), 1000.

[72] S. Garnerone, Quantum algorithms for topological invariants, PhD Thesis, Politecnico di Torino, Italy, 2007.

[73] R. K. Kaul and P. Ramadevi, Three-manifold invariants from ChernSimons field theory with arbitrary semi-simple gauge groups, Commun. Math. Phys. 217 (2001), 295.

[74] D. A. Lidar, On the quantum computational complexity of the Ising spin glass partition function and of knot invariants, New. J. Phys. 6 (2004), 167.

[75] V. Aquilanti and C. Coletti, 3nj-symbols and harmonic superposition coefficients: an icosahedral abacus, Chem. Phys. Lett. 344 (2001), 601. 